\title{
Enhanced Energy Generation and Membrane Performance by Two-stage Pressure Retarded Osmosis (PRO)
}

\author{
Wei He, Yang Wang and Mohammad Hasan Shaheed ${ }^{1}$ \\ School of Engineering and Materials Science, Queen Mary, University of London, London E1 4NS \\ E-mail:w.he@qmul.ac.uk, yanq.wang@qmul.ac.uk, m.h.shaheed@qmul.ac.uk
}

\begin{abstract}
:
A systematic model of a salinity power plant involving two PRO modules (TwoPRO) is presented in this paper. The results clearly indicate that the performance of a PRO plant can be improved with a two-stage configuration. For comparison purposes, a single-stage PRO (SinglePRO) is first analyzed and optimized with respect to three key concepts, power density, extractable energy and average power density. The two-stage configuration of the TwoPRO, is then studied using two schemes: continuous scheme in which the stream is treated as a continuous flow between the two stages and divided scheme in which the flow is divided first and separately treated in each stage. Accordingly, four different configurations are proposed, namely continuous draw and continuous feed solution (CDCF), divided draw and divided feed solution (DDDF), continuous draw and divided feed solution (CDDF), and divided draw and continuous feed solution (DDCF). The results indicate that the configurations CDCF, CDDF and DDCF have advantageous capacity in salinity energy harvesting. Configurations CDCF and CDDF also perform better than the single-stage PRO in terms of average power density with a higher feed solution flow ratio. Additionally, the membrane performance of the TwoPRO strengthens with higher level of membrane usage.
\end{abstract}

Keywords: pressure retarded osmosis, two-stage configurations, extractable energy, average power density

\section{Introduction}

Osmotic energy (or salinity power) from natural salinity gradients has been identified as a candidate renewable energy source since the 1950s [1], due to its substantial potential energy capacity, estimated to be 2 TW, which is about $13 \%$ of the current world energy consumption [2]. Research groups worldwide have investigated the feasibility of capturing energy from the mixture of freshwater and seawater by means of pressure and electrostatic driven processes, such as pressure retarded osmosis (PRO) [3-6], reverse electrodialysis [7-10], and capacitive mixing [11-16]. The concept of PRO was first illustrated by Loeb in 1975 [17]. It is an osmotic-driven membrane process that converts chemical potential developed in a mixture of water with different salinity concentration into electricity [18]. Following rapid development in the field of the specific membrane over the last decade, this technology has started to be implemented in practice. In 2009, the world's first PRO plant was launched in Norway [19, 20].

Most prior investigations to improve osmotic membrane performance are focused on quantifying, elucidating and then minimizing the performance limiting phenomena [21-26]. Over the past few

\footnotetext{
${ }^{1}$ Corresponding author: Tel: +44 (0)20 78823774

E-mail address: m.h.shaheed@qmul.ac.uk
} 
years, a significant level of improvement in water permeation and salt rejection of the membrane has been achieved [27]. In this context, the PRO technology has become more promising in practical desalination systems and salinity power plants. In fact, investigations of the PRO process have been carried out, not only as an independent power generator, but also as a pre- or post- treatment mechanisms to recover osmotic energy from the high concentration brine discharge in a hybrid process integrated with reverse osmosis and forward osmosis phenomena [28-30]. They have focused on improving transient water flux, power density of the membrane [31] and potential energy from a PRO process [32, 33]. The potential energy from the mixed seawater and freshwater can be calculated using the concept of Gibbs free energy [34]. However, the potential chemical energy represents the theoretical maximal energy involved in the mixing process, which cannot be fully used due to intrinsic thermodynamic inefficiency [4], including the frictional resistance in the transportation of water inside the membrane and the unused energy stored in the un-permeated water due to non-equilibrium mass exchange.

Well-known multi-stage configurations in the RO plant indicate that the energy cost can be reduced by adding stages in series [35]. Similarly, for a PRO process, the performance improvement in energy generation can be achieved by introducing multi-stage configurations as demonstrated in one of our previous investigations [36]. The two-stage PRO configuration is demonstrated to reduce the irreversible energy losses and increase the energy harvesting efficiency. Very recently, a dual stage PRO process is proposed and experimentally studied by Ali et al. [37]. The experimental results showed that the power generation in the dual PRO process was higher than that in the conventional PRO process. But compared with a two-stage RO configuration, more influencing factors are involved in a two-stage PRO plant, i.e. flow conditions and salinity concentration of both the draw solution and the feed solution, and the current PRO membrane performance. Therefore, more investigations are needed to focus on the possible multi-stage configurations of the PRO plant. Moreover, many of the prior studies on PRO membrane have employed a crossflow membrane test cell loaded with a small piece of flat sheet coupon [26, 38-40]. In those investigations, with such a small scale membrane, the dilution of the draw solution in the crossflow cell was considered negligible, though it could be significant as considerably large area of membrane is required in a PRO process in practice [41]. As such, the averaged power density of a larger scale PRO membrane would be substantially less than the value achieved in a laboratory scale [42]. For a large-scale PRO process with constant pressure applied, similar to the thermodynamic restriction limit in a RO process [43], due to the dilution of the draw solution and/or the concentration of the feed solution, the equilibrium of the net driving force might be reached that the water flux is reduced close to zero at the exit of the module. With such membrane usage, the overall membrane performance that is the average power density of the entire membrane used is needed to be considered.

Therefore, in this study, a comprehensive and systematic investigation is carried out on the system-based energy generation and membrane-based average power density of the possible configurations of a two-stage PRO system (TwoPRO). The system-based energy generation is the scale-independent capacity of the energy generation by a full-scale PRO process. In contrast, the membrane-based average power density is the scale-dependent average power density subject to the area of the membrane used. To carry out the study, at first, several key concepts of the PRO technology including membrane power density, scale-independent extractable energy and scaledependent average power density are respectively introduced. A single-stage PRO module (SinglePRO) is optimized to be used as a reference for comparison purposes. Furthermore, according to the flow configurations of the draw solution (DS) and the feed solution (FS), i.e. being 
continuously treated by the two stages or being divided before the first stage and treated separately by the two stages, possible configurations of the TwoPRO are investigated. It consists of four configurations, namely continuous DS and continuous FS (CDCF), divided DS and divided FS (DDDF), continuous DS and divided FS (CDDF), and divided DS and continuous FS (DDCF). All the configurations are studied from the perspective of system-based energy generation and membranebased average power density and have shown different characteristics in terms of overall performance of the system and the membrane. Finally, based on the results obtained, performance of each configuration of the TwoPRO is compared with that of the SinglePRO.

\section{Theories}

\subsection{Three key concepts}

PRO uses the natural phenomenon of osmosis to permeate water across a semi-permeable membrane from a side with low solute concentration and low hydraulic pressure to a side with high concentration and high pressure. The permeated water is then used to generate electricity in a hydro-turbine (Fig. 1). In a PRO process, the draw solution is pressurized by the pump and the energy recovery device (ERD). As water is transported across the membrane, the draw solution becomes progressively diluted and the concentration of the feed solution rises.

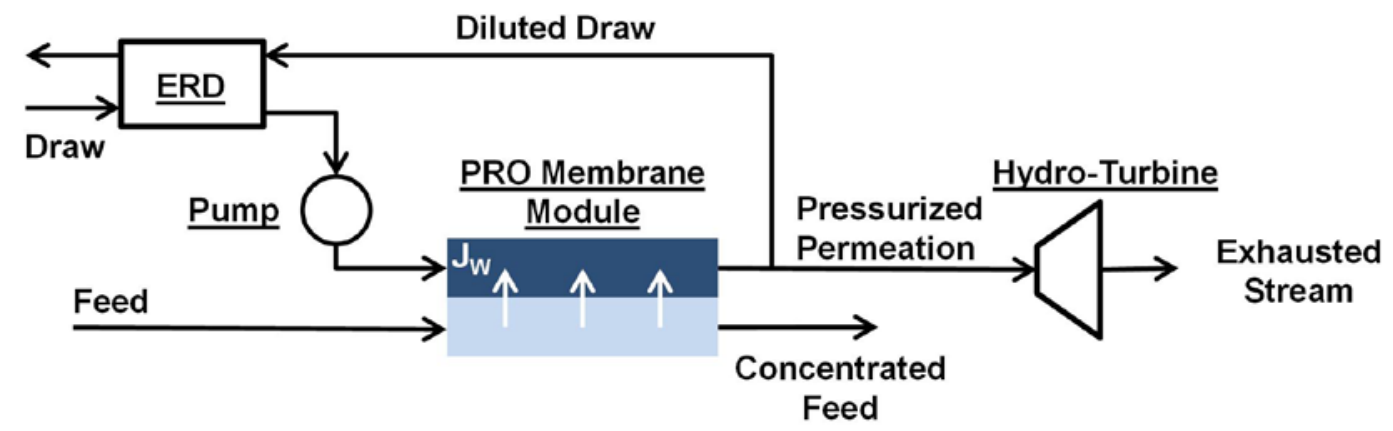

Fig. 1. A schematic illustration of a PRO process. The water permeates through the membrane from the low concentration side (feed solution) to the high concentration side (draw solution), and then is expanded in a hydro-turbine.

Theoretically, the power density of a PRO membrane is the product of the trans-membrane hydraulic pressure and the water permeation flux across the membrane. The power density can be represented as,

$$
W=J_{W} \cdot \Delta P
$$

where water flux, $J_{w}$, across the membrane is determined by,

$$
J_{W}=L_{p}\left(\Delta \pi_{m}-\Delta P\right)
$$

where $L_{p}$ is the membrane water permeability, $\Delta \pi_{m}$ and $\Delta P$ are respectively the osmotic pressure difference and the applied pressure difference between the two sides of the membrane. Based on the hypothetical solutions considered in this study, according to van't Hoff's law, the osmotic pressure difference can be represented as,

$$
\Delta \pi_{m}=\mathrm{C}_{v a n^{\prime} t}\left(\mathrm{C}_{D, m}-\mathrm{C}_{F, \mathrm{~m}}\right)
$$


where $C_{\text {van't }}$ is the modified van't Hoff coefficient, $C_{D, m}$ and $c_{F, m}$ are the concentration of the draw and the feed solution on the two sides of the membrane, respectively. The van't Hoff law is restricted to be used on dilute, ideal solution $[44,45]$. In the salinity range of $0-70 \mathrm{~g} / \mathrm{kg}$, the linear osmotic pressure approximation with the modified coefficient is validated and the maximum deviation is $6.8 \%[46,47]$.

If the concentration polarization ( $\mathrm{CP}$ ) effects are not considered, the concentration of the solution near the membrane surface is equivalent to the concentration of the bulk flows. However, the ideal situation cannot be ensured in practice due to the existence of internal concentration polarization (ICP), external concentration polarization (ECP), and reverse salt permeation (RSP). The deviation of the net driving force between the membrane surfaces and the bulk flows occurs and is determined by the membrane properties and the local water flux. Several mathematical models have been developed to represent the real concentration difference between the two sides of the membrane in terms of the bulk concentration $[6,25,48,49]$. Usually high water flux causes larger deviation and when the water flux reduces close to zero the deviation becomes negligible. The profiles of the water flux, power density and the extractable energy of a PRO plant without considering the $\mathrm{CP}$ and RSP effects are similar to the ones considering all the detrimental effects but in different quantities [50]. It indicates that the resistance in the water and solute transportation are increased due to the ICP, ECP and RSP effects and the enhanced resistance reduces the water flux across the membrane. However, on the basis of the current membrane performance, the power dynamics of the PRO plant is not significantly changed according to the similar profile of the power density and extractable energy with respect to the hydraulic pressure applied on the draw solution [50]. In addition, as the relation between the numerical models considering the detrimental effects and the ones without the consideration has been studied previously [50-52], it can be considered as the overall performance limiting effect in terms of the modeling without the ICP, ECP and RSP effects. At the early stage investigation of the two-stage PRO configuration, this work focuses on the effects of different flow configurations and the advantageous performance of the TwOPRO compared to the SinglePRO. Therefore, a model without considering the CP and RSP effects is first employed to simulate power dynamics and energy generation of the single-stage and the two-stage PRO processes.

Because of the negligible pressure drops along the membrane channels of both the draw and feed solutions, a constant hydraulic pressure is applied on the draw solution and the extractable energy can be expressed as,

$$
E_{C-P R O}=\Delta P \cdot \Delta V_{P}^{S U M}
$$

where, $E_{C-P R O}$ is represented as the system-based extractable energy from a constant-pressure PRO (C-PRO) process, $\Delta V_{p}^{S U M}$ is the volumetric rate of permeation. The concept of the extractable energy represents the full energy capacity that can be harvested applying a certain hydraulic pressure.

In fact, among the two concepts above, one describes the transient membrane behavior and the other is a state of the full-scale water transportation and neither of them appropriately represents the overall membrane performance with respect to different area scales. Therefore, average membrane power density, is derived to represent the membrane performance of a PRO process with respect to membrane area scale. It can be expressed as, 


$$
e=\frac{\int_{0}^{A_{m}^{*}} W d\left(A_{m}\right)}{A_{m}^{*}}=\frac{\left.E_{C-P R O}\right|_{A_{m}=A_{m}^{*}}}{A_{m}^{*}}
$$

where $e$ represents the average membrane power density of the membrane used, and $A_{m}^{*}$ is the scale of membrane studied.

These three concepts are illustrated in a co-current PRO process shown in Fig.2. The parameters of the process are: the applied hydraulic pressure $10 \mathrm{bar}$, membrane water permeability coefficient $1.74 \mathrm{~L} \mathrm{~m}^{-2} \cdot \mathrm{h}^{-1} \mathrm{bar}^{-1}$, the modified van't Hoff coefficient $0.7345 \mathrm{bar} \cdot \mathrm{kg} \cdot \mathrm{g}^{-1}$ [46]. For simplicity, the flow rate of the FS is assumed to be $1 \mathrm{~kg} \cdot \mathrm{h}^{-1}$. According to the methodology of the modeling, discharge behavior of the PRO process [53], the osmotic pressure differences and the applied pressure are shown in Fig. 2(a). In Fig. 2(b), the three key concepts, namely power density, average power density and the extractable average power density are presented.

Fig. 2(a), for a co-current PRO process with a constant hydraulic pressure applied, clearly indicates the net driving force in a full-scale discharge in which the equilibrium between the applied hydraulic pressure and the osmotic pressure difference is reached at the point $\mathrm{M}$. Therefore, along the flow channel, area represented by the point $\mathrm{M}$ is the minimum area required for a full-scale PRO discharge. The required membrane area is dependent on the flow rates of both the DS and FS. With different flow rates, the required membrane area also varies. In Fig. 2(b), it is observed that without consideration on the RSP effects, when the balance of the hydraulic pressure and the osmotic pressure difference is built, the local power density of the membrane is reduced to zero because of the equilibrium of the net driving force and the extractable energy stops to increase. Additionally, the average power density starts at the value overlapping with the local power density with the limiting zero area of the membrane. Along the flow channel with the increase of the membrane usage, the average power density keeps decreasing but all values are higher than the transient local power density. Profiles of the three concepts are illustrated by the marked three points (point $A, B$ and $\mathrm{C}$ ) in Fig. 2(b) when the membrane area is less than the minimum area (point $M$ ) required for a full-scale PRO discharge. The local membrane power density is the transient property of the local channel position, represented by point $A$. The accumulating extractable energy is denoted by point $C$ and the average power density of the overall membrane is denoted by point $B$. 


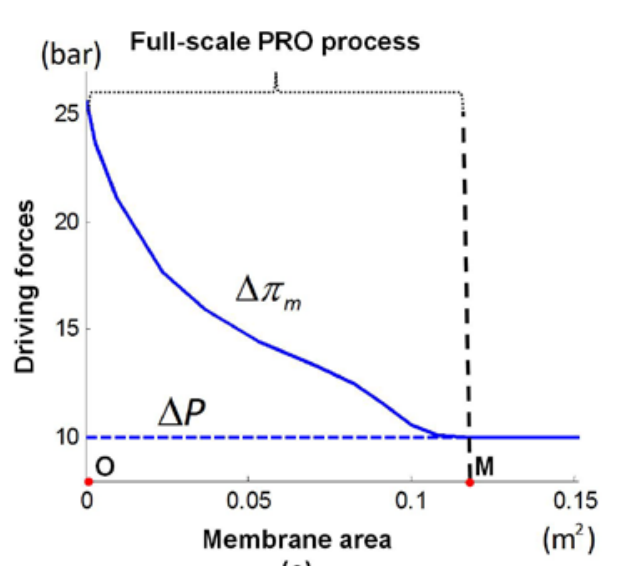

(a)

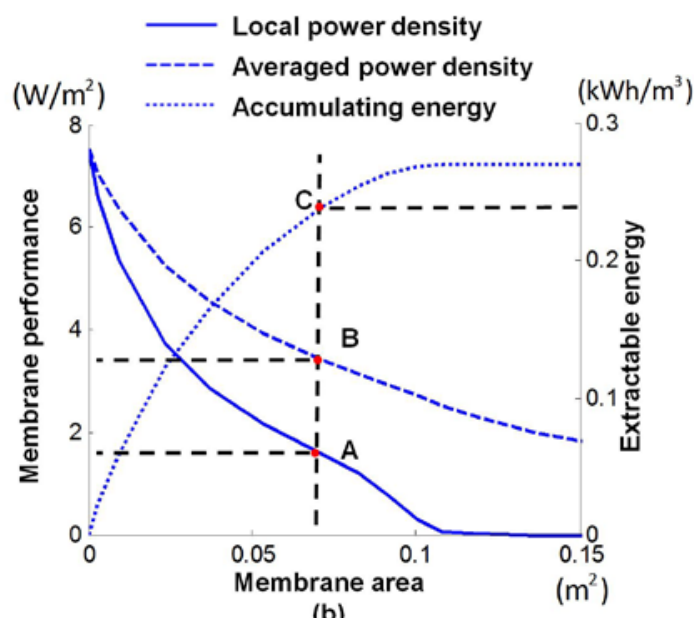

(b)

Fig. 2. Illustration of the three key concepts in a PRO process. In (a), the driving forces of the mass exchange is presented, including both hydraulic pressure and the osmotic pressure differences along the membrane channels. And the local and the average power density are illustrated in (b).

\subsection{Comparison between the three key concepts in a SinglePRO}

With certain salinity gradients available in a SinglePRO process as illustrated in Fig. 1, the most influential factor is the hydraulic pressure on the DS. It controls the water permeation across the membrane and then results in the variation in salinity power harvest. Different optimization objectives drive different process operation resulting in different performance of the PRO. The variation of the membrane power density and system-based extractable energy with respect to the applied pressure on the draw solution, are illustrated in Fig. 3(a) and (b), respectively. The parameters used in simulation are: DS $35 \mathrm{~g} / \mathrm{kg}$ seawater, and FS $0.1 \mathrm{~g} / \mathrm{kg}$ freshwater. On the basis of the results, with different dimensionless flow ratios, the unit membrane power densities are same but the system-based energy capacity is different. In fact, according to Equations (1), and (4), the optimum applied pressure of the membrane power density, $\Delta P_{O P T}^{P D}$, and the optimum of the systembased extractable energy, $\Delta P_{O P T}^{C-P R O}$, can be obtained by setting $d W / d(\Delta P)=0$ and $d E_{C-P R O} / d(\Delta P)=0$, respectively [4]. And the results are shown in Fig. 3(c). The results clearly indicate that the optimal pressure of the membrane power density is independent of the flow rate of the DS and FS. Conversely, the optimal pressure for the maximum extractable energy is inversely proportional to the dimensionless flow rate. 

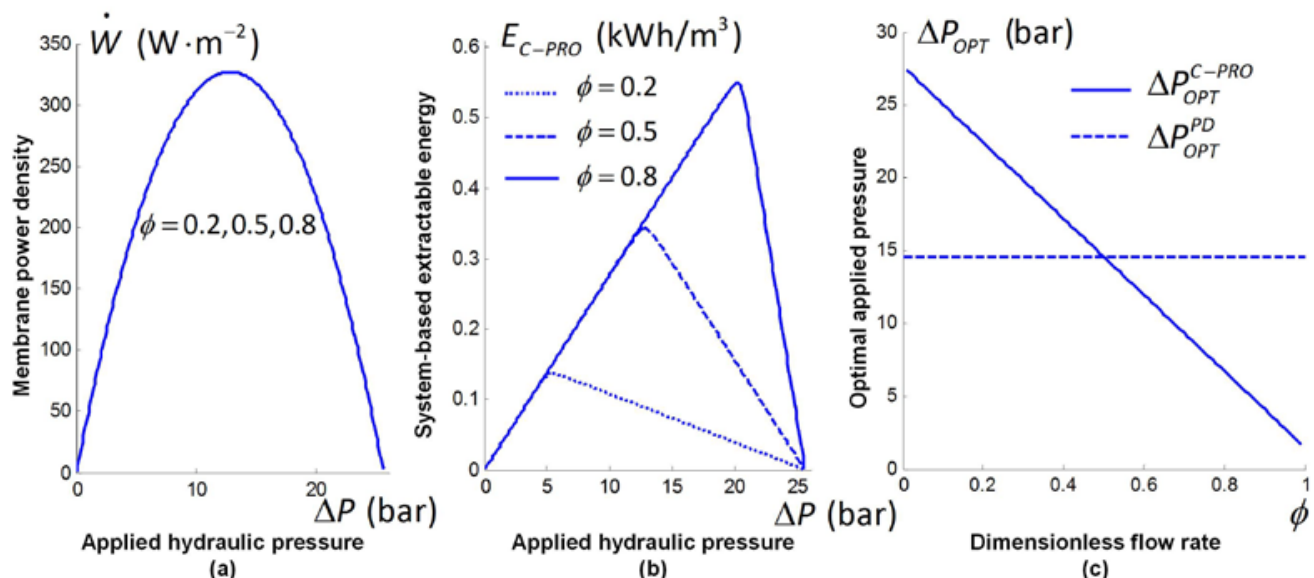

Fig. 3. Variation of the membrane power density (a) and system-based extractable energy (b) with respect to different values of the hydraulic pressure applied on the draw solution. In (c), the optimum applied pressure to achieve the maximum power density and the C-PRO extractable energy are presented, respectively.

Furthermore, based on Equation (5), it is known that with the increase of the membrane area, the average power density keeps decreasing due to the diluting DS and the concentrating FS. However, with a certain area of the membrane used, the average power density can be controlled by applying different magnitude of the hydraulic pressure on the DS. In a recent study carried out by Banchik et al., the optimum applied pressure difference for the maximum average power density of a countercurrent PRO process is studied based on the $\varepsilon$-MTU model [54]. In a co-current flow scheme, for the same target aimed at using the model above, the optimization problem can be mathematically represented as,

$$
\begin{array}{ll}
\max \quad & \left.e(\Delta \mathrm{P})\right|_{A_{m}^{*}}=\frac{\Delta P \int_{0}^{A_{m}^{*}} L_{P}\left(C_{\text {van't }}\left(\frac{1-\phi}{1-\phi\left(1-\Delta q_{P} / q_{F}^{0}\right)} C_{D}^{0}-\frac{1}{1-\Delta q_{P} / q_{F}^{0}} C_{F}^{0}\right)-\Delta P\right) d A_{m}}{A_{m}^{*}} \\
\text { subject to } \quad 0 \leq \mathrm{A}_{m}^{*} \leq \mathrm{A}_{m}^{F U L L} ; 0 \leq \Delta P \leq \Delta P^{M A X}
\end{array}
$$

where $A_{m}^{\text {FULL }}$ is the minimum membrane area required by the full-scale water permeation. In accordance with Equation (6), all the operation with different applied pressure were evaluated and compared. Then, the optimum pressure with respect to the membrane area is obtained. As the average power density is dependent not only on the applied pressure but also on the membrane area and the availability of the salinity streams, the optimum applied pressure of the average power density is studied with respect to three dimensionless flow ratio, $0.2,0.5$ and 0.8 . The membrane water permeability is $2 \mathrm{~L} \mathrm{~m}^{-2} \cdot \mathrm{h}^{-1} \mathrm{bar}^{-1} .1 \mathrm{~kg} / \mathrm{h}$ feed flow rate of the FS is assumed for simplicity.

The optimization results with respect to average power density are shown in Fig. 4. In (a), the optimum average power densities at different membrane area scales are presented. The average power density is influenced by the flow rates of the salinities. The PRO with lower dimensionless flow rate results in higher average power density at all scales of the membrane area. In addition, for a certain dimensionless flow rate, the potential maximum of average power density keeps decreasing with the increase of the membrane area. Based on the results in (b), it is clear that the optimal pressure of the average power density is also area-dependent. 

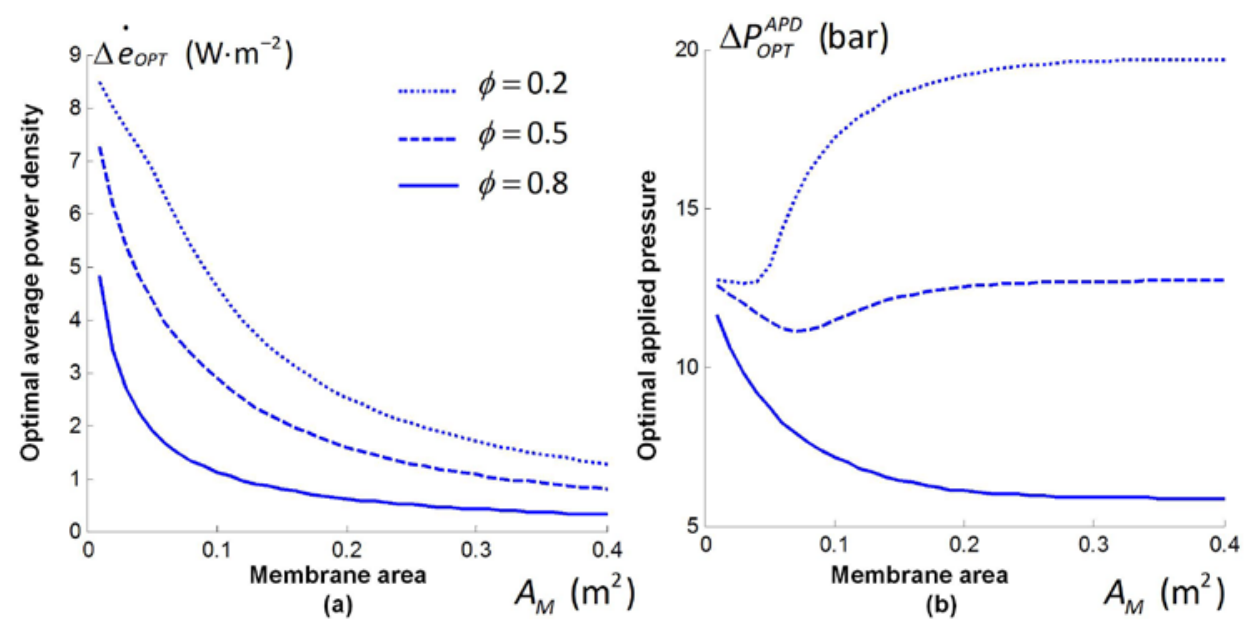

Fig. 4. The optimization of the SinglePRO in terms of average power density. In (a) and (b), the optimum pressure and the maximum average power density are presented, respectively. The flow rate of the FS is assumed to be $1 \mathrm{~kg} \cdot \mathrm{h}^{-1}$.

\section{Performance of Two-PRO systems}

Therefore, based on the results of the local power density, the extractable energy and the average power density, it is clearly indicated that these three concepts are inherently different. Usually, the operation with different objectives is not compatible. In other words, it is difficult to achieve the both/all maximums of the three targets simultaneously in a SinglePRO. Furthermore, in a TwoPRO plant, more interactions between the two stages are involved and the optimum performance and the required operation become complicated. Therefore, a systematic investigation of the optimum performance needs to be carried out. According to the performance of the SinglePRO, the power density is the performance of the local membrane unit, the extractable energy presents the performance of the full-scale PRO process and the average power density denotes the overall membrane performance of the membrane used. In order to study the process performance of the possible TwoPRO configurations, the extractable energy and the average power density are selected as the objectives to compare between the TwoPRO and the SinglePRO at a later stage.

In the investigation of the TwoPRO, a SinglePRO is the base element in all the PRO applications in this study. Because this study aims to investigate the differences and the potential advantages of the different TwoPRO configurations with respect to a SinglePRO process, for simplicity the co-current flow scheme is first considered at the early stage of the TwoPRO. Thus, each sub-PRO module in a TwoPRO has the same configuration as the SinglePRO illustrated in Fig. 1. And thus each of them performs strictly in the same principle as the SinglePRO, including all the membrane power density, system-based extractable energy, average power density, and the operations. Therefore, the emphasis to investigate the TwoPRO is to find the advantages and the disadvantages of the TwoPRO over the extractable energy and the average power density.

\subsection{Two-PRO configurations}

According to different flow schemes between the two PRO stages in a TwoPRO, four configurations are defined. They are CDCF, DDDF, CDDF, and DDCF, as illustrating in Fig. 5 in which ' $D$ ' and ' $F$ ' in each PRO module represents the DS and FS flow channel, respectively. Among the configurations, ' $C$ ' is shortened for the continuous treatment which means the solution is treated continuously by the two stages. For instance, in the configuration CDCF, both the DS and the FS are continuously treated by the two stages that the solution from the outlets of the first stage are the 
solution as the initial salinities for the second stage. In contrast, ' $D$ ' is shortened for the divided treatment in which the solution is divided before it flows into the first stage and treated separately in each stage. For example, in the configuration DDCF, the DS solution is divided into two branches and flows into the two stages separately as the high concentration solution.

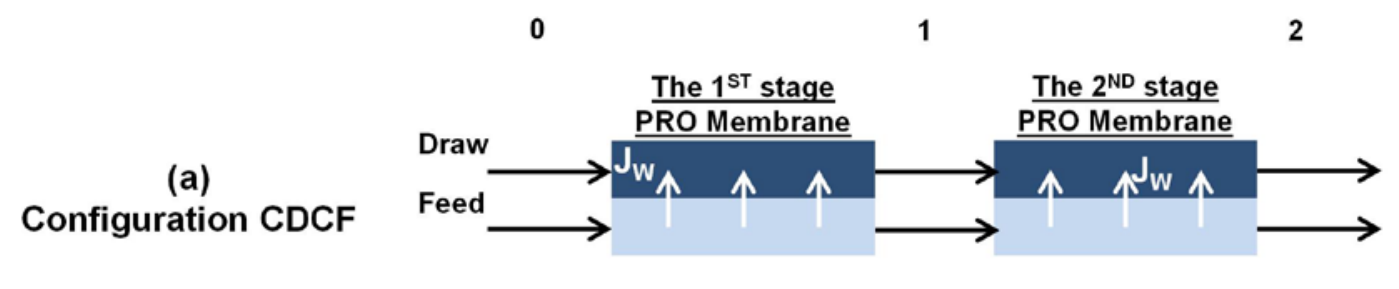

(b)

Configuration DDDF

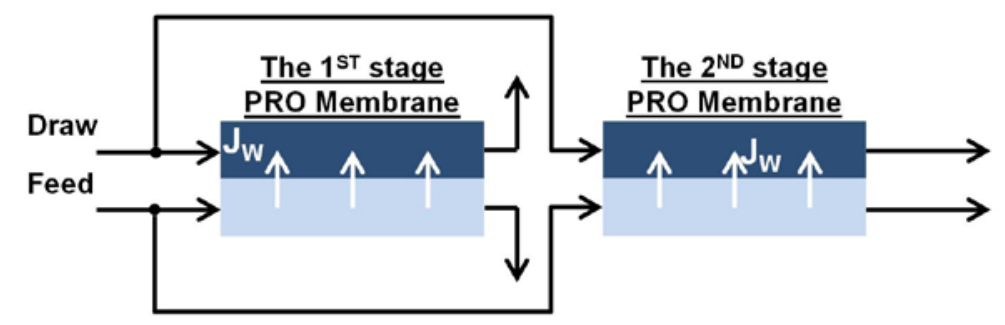

(c)

Configuration CDDF

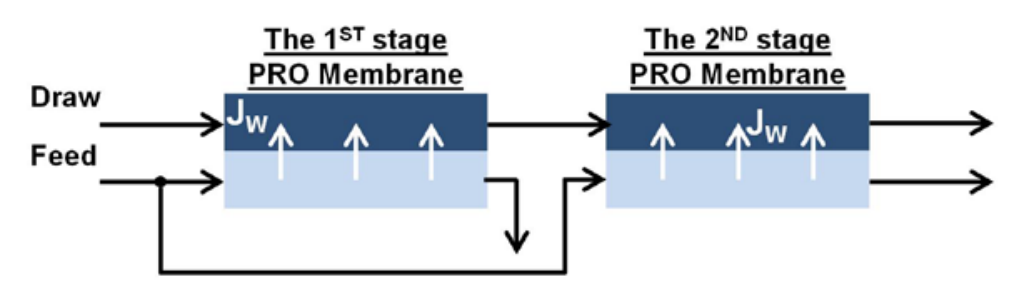

(d)

Configuration DDCF

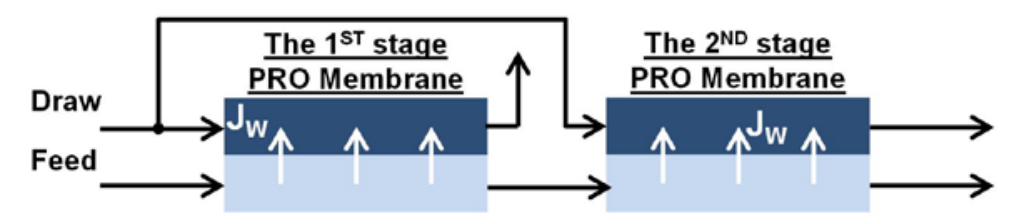

Fig. 5. Schematic diagram of the four possible configurations of the TwoPRO process. In the figure, number 0 (inlet state of the first stage), 1 (outlet state of the first stage or inlet state of the second stage) and 2 (outlet state of the second stage) denote different process state of the water treatment.

In a TwoPRO system, several new parameters appear to be considered. They are flow rate distribution ratio of the DS and the FS, and the membrane area allocation ratio. For purpose of comparing with the SinglePRO, the same salinity gradients and the same amount of the membrane area are used in a TwoPRO system. The efficiency of the energy extraction can be compared between the single-stage and two-stage configurations by using the same salinities. With the same initial draw and feed solutions, more energy generation from a certain configuration means a higher efficiency is achieved in the salinity energy harvest. In addition, to evaluate the overall membrane performance, average power density is used. Because the scale-dependence of the average power density, the same amount of the membrane usage is to guarantee the validated comparisons between the SinglePRO and the TwoPRO in terms of the average power density. Moreover, the 
hydraulic pressure applied on the draw solution in the two modules is still a crucial operational parameter.

\subsection{System state of TwoPRO - scale-independent extractable energy}

\subsubsection{Mathematic models}

Similar to the optimization in the SinglePRO system, the total maximum extractable energy is the main target to evaluate the system-based performance. The maximums of the extractable energy are searched among the different distribution flow ratios of the DS, and the $F S,\left(D F_{D}^{1 s t}, D F_{D}^{2 n d}\right)$ and $\left(D F_{F}^{1 s t}, D F_{F}^{2 n d}\right)$, and the allowable applied hydraulic pressures in the two PRO modules, $\Delta P^{1 s t}$ and $\Delta P^{2 n d}$.

For all the four configurations, based on the mass balance, the sum of the distribution ratios of both the DS and the FS, should be unity, which is,

$$
\begin{aligned}
& D F_{K}^{1 s t}+D F_{K}^{2 n d}=1 ; \\
& q_{K}^{1 s t}=D F_{K}^{1 s t} q_{K}^{0} ; q_{K}^{2 n d}=D F_{K}^{2 n d} q_{K}^{0} ;
\end{aligned}
$$

where the subscript $k$ denotes the DS or the FS in flow distribution. With different flow distribution ratios of the DS or/and the FS, the dimensionless flow ratios are different. The two dimensionless ratio in the first stage can be expressed in terms of the $D F_{D}^{1 s t}$ and $D F_{F}^{1 s t}$ as follows,

$$
\phi^{1 s t}=\frac{D F_{F}^{1 s t} \phi^{0}}{D F_{F}^{1 s t} \phi^{0}+D F_{D}^{1 s t}\left(1-\phi^{0}\right)}
$$

where $\phi^{0}$ represents the initial dimensionless flow ratio that is the same as the dimensionless flow ratio of the SinglePRO, and $\phi^{1 \text { st }}$ denotes the dimensionless flow rate in the first PRO module. For the second PRO module, with different flow patterns, dimensionless flow rate $\phi^{2 n d}$ varies.

The inlet condition of the second PRO module is dependent on the outlet condition of the DS and the FS from the first PRO module and the configurations. After the treatment in the first PRO module, the DS becomes diluted and the FS is concentrated due to a certain amount of water transportation from the FS to the DS. Therefore, the concentration of the DS and the FS at the outlet of the first PRO module can be represented as,

$$
c_{D}^{1}=\frac{c_{D}^{0} q_{D}^{1 s t}}{q_{D}^{1 s t}+\Delta q_{P}^{1 s t}} ; c_{F}^{1}=\frac{c_{F}^{0} q_{F}^{1 s t}}{q_{F}^{1 s t}-\Delta q_{P}^{1 s t}}
$$

where the superscript 1 represents the state of the solutions after the treatment of the first PRO module, and $\Delta q_{p}^{1 s t}$ is the mass flow rate permeated in the first PRO module. In the extractable energy modeling, without area consideration, enough membrane is assumed for the full-scale water permeation (area of the membrane used is no less than the minimum required area), that the maximum salinity energy based on the constant pressure PRO extraction is considered. In such an 'area-free' condition in which the extractable energy is area-independent, the mass flow rate in the first stage can be further written by setting $d E_{C-P R O} / d\left(\Delta V_{\text {SUM }}\right)=0$ [4], 


$$
\Delta q_{P}^{1 s t}=\frac{\sqrt{c_{D}^{0}}-\sqrt{c_{F}^{0}}}{\sqrt{c_{D}^{0}}+\frac{\phi^{1 s t}}{1-\phi^{1 s t}} \sqrt{c_{F}^{0}}} q_{F}^{1 s t}
$$

For the configurations with continuous FS or/and DS flow scheme, the flow rates' variation in the second PRO module also depend on the rate of the permeated water in the first module,

$$
\Delta q_{D}^{2 n d}=q_{D}^{1 s t}+\Delta q_{P}^{1 s t} \text { or/and } \Delta q_{F}^{2 n d}=q_{F}^{1 s t}-\Delta q_{P}^{1 s t}
$$

Therefore, the overall extractable energy of a TwoPRO system can be expressed as,

$$
E_{\text {TwoPRO }}=E_{C-P R O}^{1 s t} \times D F_{F}^{1 s t}+E_{C-P R O}^{2 n d} \times\left(D F_{F}^{2 n d}+\frac{\Delta q_{F}^{2 n d}}{q_{F}^{0}}\right)
$$

For different configurations, the relation between the two modules with respect to these variables is different. The models derived for simulating the extractable energy in the four configurations are listed in Table 1 . In the table, $c_{D}^{2 n d, 0}$ and $c_{F}^{2 n d, 0}$ denote the initial concentration of the draw and feed solution in the second stage, respectively.

\begin{tabular}{|c|c|c|c|c|c|}
\hline $\begin{array}{l}\text { Confi } \\
\text { gurati } \\
\text { on }\end{array}$ & $\begin{array}{l}\text { Distribution } \\
\text { ratio of the } \\
\text { DS }\end{array}$ & $\begin{array}{c}\text { Distribution } \\
\text { ratio of the } \\
\text { FS }\end{array}$ & $\begin{array}{c}\text { Concentration } \\
\text { of the DS and } \\
\text { the FS before } \\
\text { the second } \\
\text { PRO module }\end{array}$ & $\begin{array}{l}\text { Applied hydraulic } \\
\text { pressures in the two } \\
\text { PRO modules }\end{array}$ & $\begin{array}{l}\text { Flow rates' } \\
\text { variations in the } \\
\text { second PRO } \\
\text { module }\end{array}$ \\
\hline \multirow[t]{2}{*}{$\mathrm{CDCF}$} & $D F_{D}^{1 s t}=1$ & $D F_{F}^{1 s t}=1$ & $c_{D}^{2 n d, 0}=c_{D}^{1}$ & $0 \leq \Delta P^{1 s t} \leq C_{\text {van }, t}\left(c_{D}^{0}-c_{F}^{0}\right)$ & $\Delta q_{D}^{2 n d} \neq 0$ \\
\hline & $D F_{D}^{2 n d}=0$ & $D F_{F}^{2 n d}=0$ & $c_{F}^{2 n d, 0}=c_{F}^{1}$ & $0 \leq \Delta P^{2 n d} \leq C_{v a n, t}\left(c_{D}^{1}-c_{F}^{1}\right)$ & $\Delta q_{F}^{2 n d} \neq 0$ \\
\hline \multirow[t]{2}{*}{ DDDF } & $0<D F_{D}^{1 s t}<1$ & $0<D F_{F}^{1 s t}<1$ & $c_{D}^{2 n d, 0}=c_{D}^{0}$ & $0 \leq \Delta P^{1 s t} \leq C_{v a n, t}\left(c_{D}^{0}-c_{F}^{0}\right)$ & $\Delta q_{D}^{2 n d}=0$ \\
\hline & $0<D F_{D}^{2 n d}<1$ & $0<D F_{F}^{2 n d}<1$ & $c_{F}^{2 n d, 0}=c_{F}^{0}$ & $0 \leq \Delta P^{2 n d} \leq C_{v a n, t}\left(c_{D}^{0}-c_{F}^{0}\right)$ & $\Delta q_{F}^{2 n d}=0$ \\
\hline \multirow[t]{2}{*}{ CDDF } & $D F_{D}^{1 s t}=1$ & $0<D F_{F}^{1 s t}<1$ & $c_{D}^{2 n d, 0}=c_{D}^{1}$ & $0 \leq \Delta P^{1 s t} \leq C_{\text {van }, t}\left(c_{D}^{0}-c_{F}^{0}\right)$ & $\Delta q_{D}^{2 n d} \neq 0$ \\
\hline & $D F_{D}^{2 n d}=0$ & $0<D F_{F}^{2 n d}<1$ & $c_{F}^{2 n d, 0}=c_{F}^{0}$ & $0 \leq \Delta P^{2 n d} \leq C_{v a n, t}\left(c_{D}^{1}-c_{F}^{0}\right)$ & $\Delta q_{F}^{2 n d}=0$ \\
\hline \multirow[t]{2}{*}{ DDCF } & $0<D F_{D}^{1 s t}<1$ & $D F_{F}^{1 s t}=1$ & $c_{D}^{2 n d, 0}=c_{D}^{0}$ & $0 \leq \Delta P^{1 s t} \leq C_{\text {van }, t}\left(c_{D}^{0}-c_{F}^{0}\right)$ & $\Delta q_{D}^{2 n d}=0$ \\
\hline & $0<D F_{D}^{2 n d}<1$ & $D F_{F}^{2 n d}=0$ & $c_{F}^{2 n d, 0}=c_{F}^{1}$ & $0 \leq \Delta P^{2 n d} \leq C_{v a n, t}\left(c_{D}^{0}-c_{F}^{1}\right)$ & $\Delta q_{F}^{2 n d} \neq 0$ \\
\hline
\end{tabular}

Table 1

Parameters of the four configurations of the TwoPRO system.

\subsubsection{Simulation and Results}

The osmotic pressure in this study is dependent on the modified van't Hoff law presented in [55] that the modified osmotic coefficient is $0.07307 \mathrm{bar} \cdot \mathrm{kg} \cdot \mathrm{g}^{-1}$. The range of the initial dimensionless flow rate is from 0.2 to 0.8 , representing different volume of the salinity gradients available. And the flow rate distribution ratio ranges from 0 to 1 . The flowchart of the simulating extractable energy in 
the TwoPRO is illustrated in Fig. 6. The extractable energy of the each configuration with all the possible flow distributions and applied pressures are modeled. The used controlled parameters in the simulation, namely the flow distributions and the applied pressures, start at the minimums and increase to the maximums on the basis of the selected step sizes. After comparing all the possible values of the system-based energy capacity, the optimums at different operation and water condition are obtained.

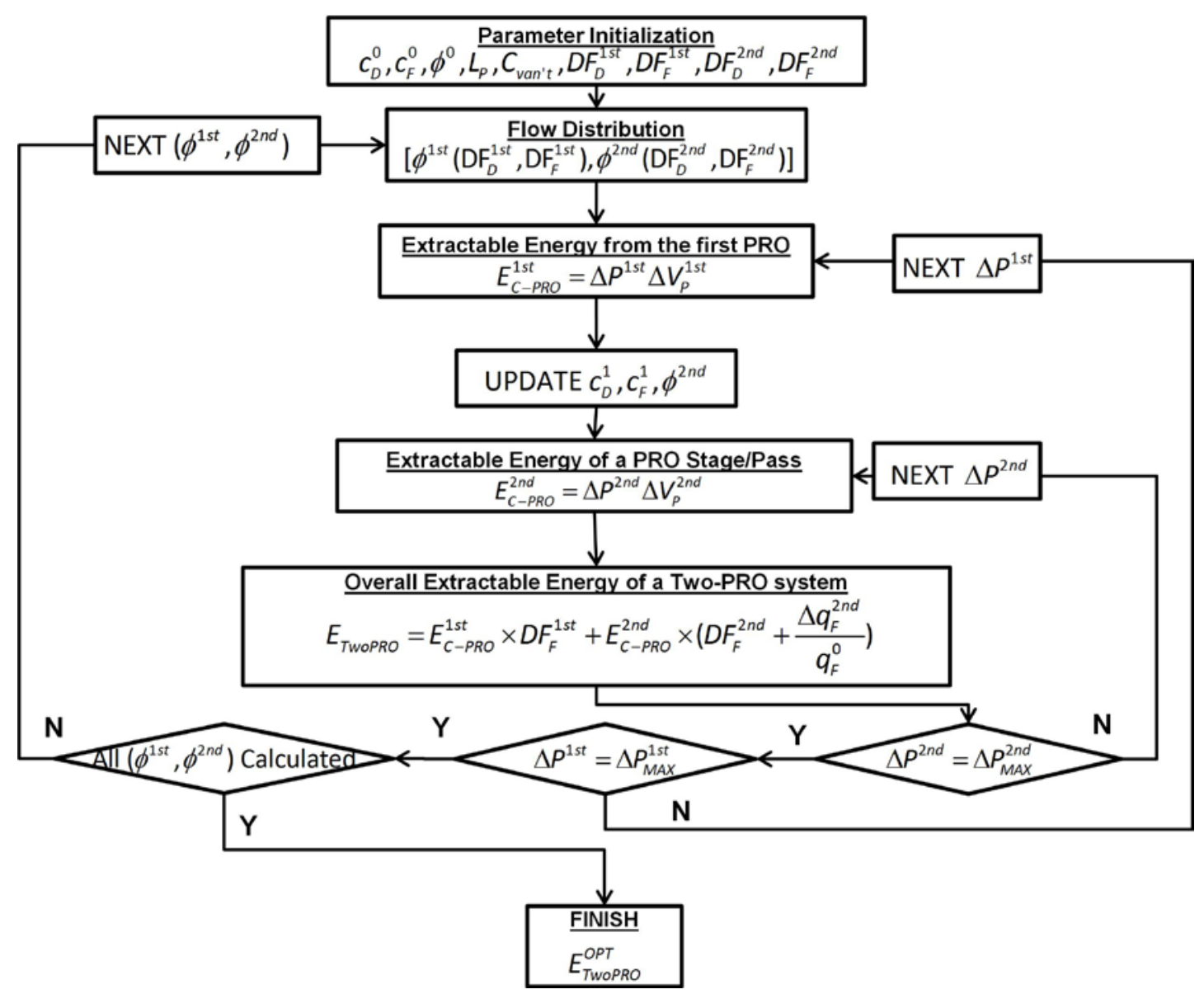

Fig. 6. The flowchart of the optimization in terms of the system-based extractable energy of the four TwoPRO configurations.

In the case of CDCF, both the FS and the DS are series connected and the salinity gradients are continuously treated in two stages. The maximum extractable energy of the configuration CDCF is illustrated in Fig. 7(a). The dotted line represents the maximum extractable energy that can be generated from the SinglePRO. Based on the results, the energy capacity of the configuration CDCF is higher than that of the SinglePRO in all the range of the dimensionless flow rate. Fig. 7(b) shows that for the higher energy generation from CDCF, higher hydraulic pressure is also required, especially for the first PRO module. In such a case, the mechanical strength of the membrane is needed to be considered [56]. Furthermore, the energy surplus is considered and is defined as follows,

$$
\Delta E_{C-P R O}=E_{\text {TWOPRO }}^{\text {MAX }}-E_{\text {SinglePRO }}^{M A X}
$$


where $E_{\text {TwoPRO }}^{\text {MAX }}$ and $E_{\text {SinglePRO }}^{\text {MAX }}$ represent the maximum C-PRO energy extraction from the TwoPRO and the SinglePRO respectively at the same dimensionless water ratio. The same concept of energy surplus is used in the later analysis in other configurations. The energy surplus of the CDCF is presented in Fig. 7(c). It is noted that although CDCF has advantageous energy capacity in all dimensionless flow rate, but its magnitudes are different with respect to the dimensionless flow rate. The maximum energy surplus of the configuration CDCF is reached between the dimensionless flow ratio 0.5 and 0.6 .

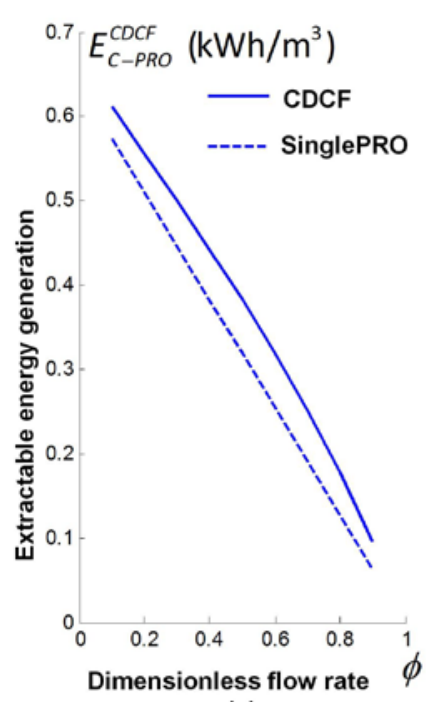

(a)

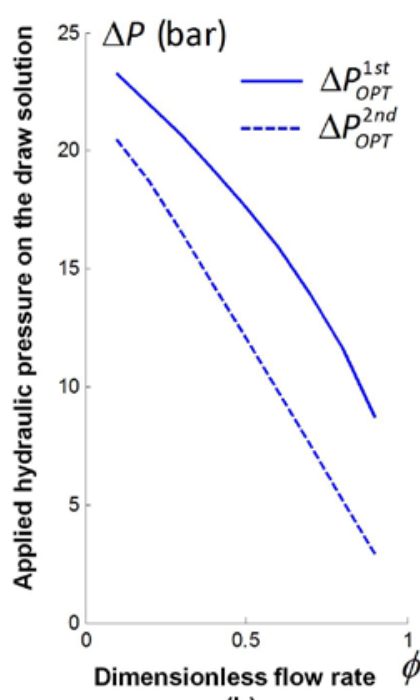

(b)

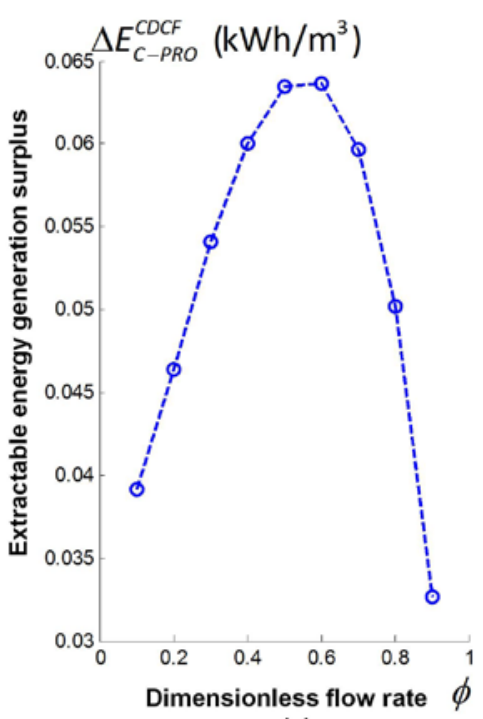

(c)

Fig. 7. System-based extractable energy of the configuration CDCF. In (a), (b) and (c), the maximum C-PRO extractable energy, optimum hydraulic pressure and the energy surplus are presented, respectively.

In configuration DDDF, two streams of the DS and the FS are divided the beginning and are treated separately by the two PRO modules. Therefore, both the DS and FS flow distribution ratios need to be considered. The extractable energy surplus is presented in Fig. 8. The energy capacity of the configuration DDDF is less than that of the SinglePRO in all the possible operations. It is noted that the negative optimum energy surpluses are found in all the distributions of the feed and draw flow among the three dimensionless flow rates $0.2,0.5$ and 0.8 . Therefore, the performance of the configuration DDDF is worse than that of the single-stage PRO plant in terms of extractable energy. But with the larger dimensionless flow ratio (0.8), the energy surplus approaches closer to zero that the extractable energy of the configuration DDDF is close to the energy of the SinglePRO. 
(a)

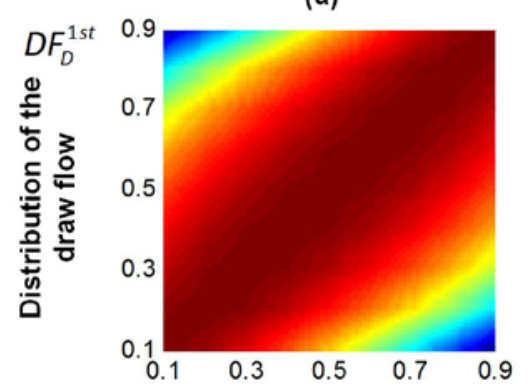

Energy surplus of the configuration DDDF $\Delta E_{C-P R O}^{D D D F}\left(\mathrm{kWh} / \mathrm{m}^{3}\right)$

(b)

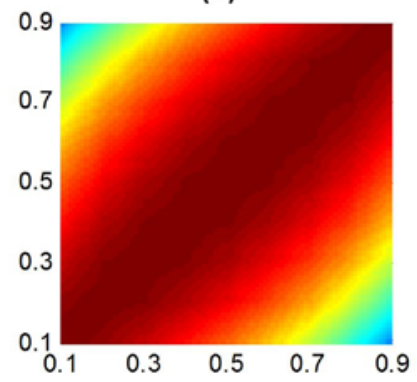

(c)

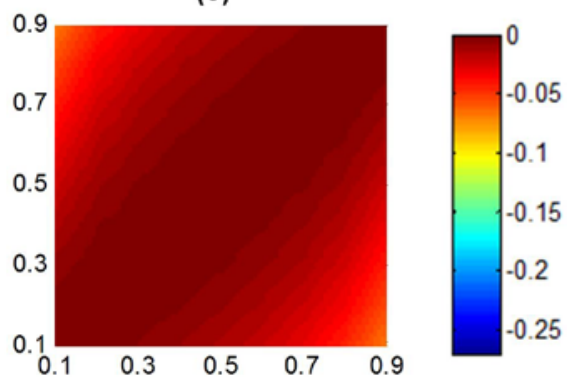

Distribution of the feed flow $D F_{F}^{1 s t}$

Fig. 8. Energy surpluses of the system-based extractable energy of the configuration DDDF in TwoPRO. The dimensionless flow rates of Fig. 8(a), (b) and (c) are $0.2,0.5$ and 0.8 , respectively.

In the configurations CDDF and DDCF, only one stream, either the DS or the FS needs to be considered in the flow distribution. The results of these two configurations are shown in Fig. 9. In Fig. 9(a), the energy surplus of the configuration CDDF is positive indicating its advantageous salinity power harvesting potential. And with a fixed dimensionless flow rate, each energy curve has a maximum. Compared with the curves with different dimensionless flow rates, the optimum dimensionless flow ratios are different. Conversely, results presented in Fig. 9(b), indicate that a larger dimensionless flow rate in DDCF results in a lower energy surplus over the SinglePRO. Further comparing the two configurations, it can be seen that the maximum energy surplus of the CDDF occurs at the lower range of flow distribution rates of the first module, i.e. $0 \leq D F_{F}^{1 s t} \leq 0.5$. In contrast, the maximum energy surplus of the configuration DDCF occurs at the higher flow distribution rates of the first module, i.e. $0.5 \leq D F_{D}^{1 s t} \leq 1$.

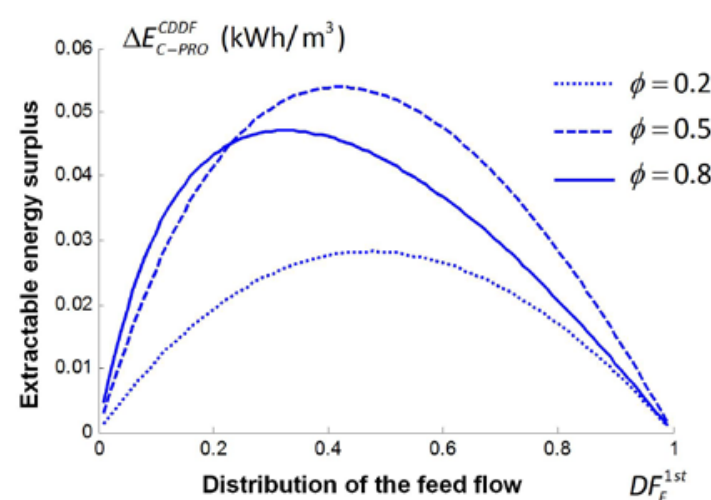

(a)

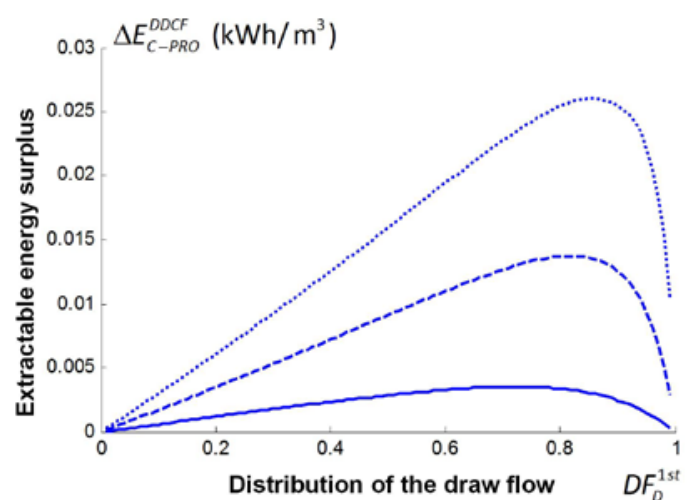

(b)

Fig 9. Optimization of the system-based extractable energy of the configurations CDDF and DDCF. In (a) and (b), the optimal energy surplus from all the operations of the hydraulic pressure are presented, respectively.

The energy surplus maxima of these two configurations are presented in Fig. 10. The relation between the maximum energy surplus and the dimensionless flow ratio is demonstrated more explicitly in these two configurations. 


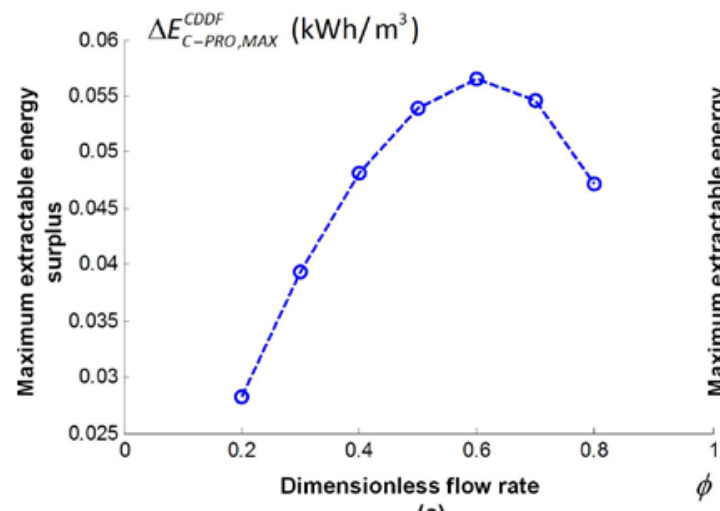

(a)

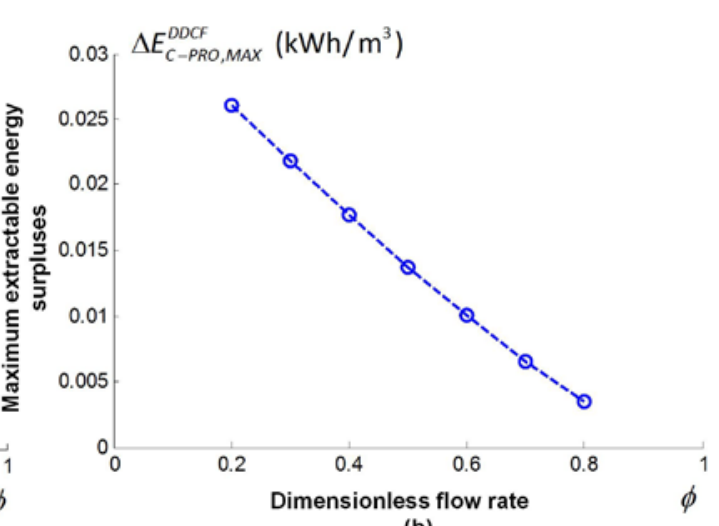

(b)

Fig. 10. Energy surplus maxima with respect to different dimensionless flow rates generated by the configurations CDDF (a) and configuration DDCF (b).

In CDDF, according to the profile of maximum energy surpluses, the highest energy extraction occurs around dimensionless flow ratio 0.6 . Conversely, the maximum energy surplus caused by the DDCF is inversely proportional to the dimensionless flow rate. Larger rates results in lower values of maximum energy surpluses. It is noted that the maximum energy surplus from the two configurations at the dimensionless flow rate 0.2 has similar value. Considering the characteristics discussed above, the configuration CDDF is preferred from the system-based energy capacity view point.

\subsection{Membrane performance of TwoPRO - scale-dependent average power density}

Analysis on the scale-independent extractable energy is an evaluation of the energy potential of the full-scale PRO process. However, if the less area of the membrane is used in a plant, the process performance is scale-dependent. Therefore, a scale-dependent concept is needed in such level of membrane usage. In this section, the average power density is used to study the performance of the PRO plant that the area used is less than the required minimum area of a full-scale process.

\subsubsection{Mathematical modeling}

In the simulation of the average power density, in addition to the flow distribution ratio and the applied hydraulic pressure in both modules, the membrane area ratio should also be considered. Due to the scale-dependence of the average power density, for purpose of comparison, the total membrane area used in each configuration of the TwoPRO is the same as the membrane area used in the SinglePRO. The membrane allocation ratios in the two stages of TwoPRO can be represented by $m^{1 s t}$ and $m^{2 n d}$, respectively, which satisfies,

$$
\begin{aligned}
& m^{1 s t}+m^{2 n d}=1 ; \\
& A_{M}^{1 s t}=m^{1 s t} A_{M}^{\text {SinglePRo }} ; A_{M}^{2 n d}=m^{2 n d} A_{M}^{\text {SinglePRO }}
\end{aligned}
$$

where $A_{M}^{1 s t}$ and $A_{M}^{2 \text { nd }}$ are the membrane area used in the first and the second PRO module respectively, and $A_{M}^{\text {SinglePRO }}$ is the membrane area used in the SinglePRO modeling.

Another crucial difference of the modeling using the average power density concept is the flow of the permeated water in the first PRO module, $\Delta q_{p}^{1 s t}$. Without the 'area-free' assumption mentioned 
in Section 3.2, the permeation in the first stage significantly depends on the membrane area allocated to the first module. Therefore, it can be rewritten as,

$$
\Delta q_{P, M}^{1 s t}=\int_{0}^{A_{M}^{1 s t}} \rho J_{W} d\left(A_{M}\right)
$$

where $\Delta q_{P, M}^{1 s t}$ denotes the changed permeated flow rate considering the membrane area usage, $\rho$ is the density of the permeation. The density of the permeation is dependent on the concentration of the solution. The empirical correlation of the density from [57] is used in this study.

Therefore, the average power density of the TwoPRO can be expressed in terms of flow distribution and the membrane area allocation as,

$$
e_{\text {TWOPRO }}=\frac{e_{1 s t} \times \mathrm{A}_{M}^{1 s t} \times D F_{F}^{1 s t}+e_{2 n d} \times \mathrm{A}_{M}^{2 n d} \times\left(D F_{F}^{2 n d}+\frac{\Delta q_{F}^{2 n d}}{q_{F}^{0}}\right)}{A_{M}^{1 s t}+A_{M}^{2 n d}}
$$

\subsubsection{Simulation and results}

In the simulation of the average power density of the TwoPRO systems, a relative average power density surplus is used which is defined as,

$$
r=\frac{\Delta e_{\text {TwoPRO }}-\Delta e_{\text {SinglePRO }}}{\Delta e_{\text {SinglePRO }}}
$$

where $\Delta e_{\text {singlepro }}$ refers to the maximum average power density achieved in the SinglePRO with respect to different membrane area. The scale of area considered is $0.1,0.2,0.3$ and $0.4 \mathrm{~m}^{2}$, allowable for the PRO operation from the limiting zero water permeation to the full-scale water permeation with the initial feed flow rate $1 \mathrm{~kg} / \mathrm{h}$. The process to find the maximum average power density is similar to that of the system-based energy capacity, but with consideration of the membrane area allocation. The process is illustrated using a flowchart in Fig. 11. In the simulation, all the possible operations are modeled and compared for the optimal average power density of the each configuration of the TwoPRO. 


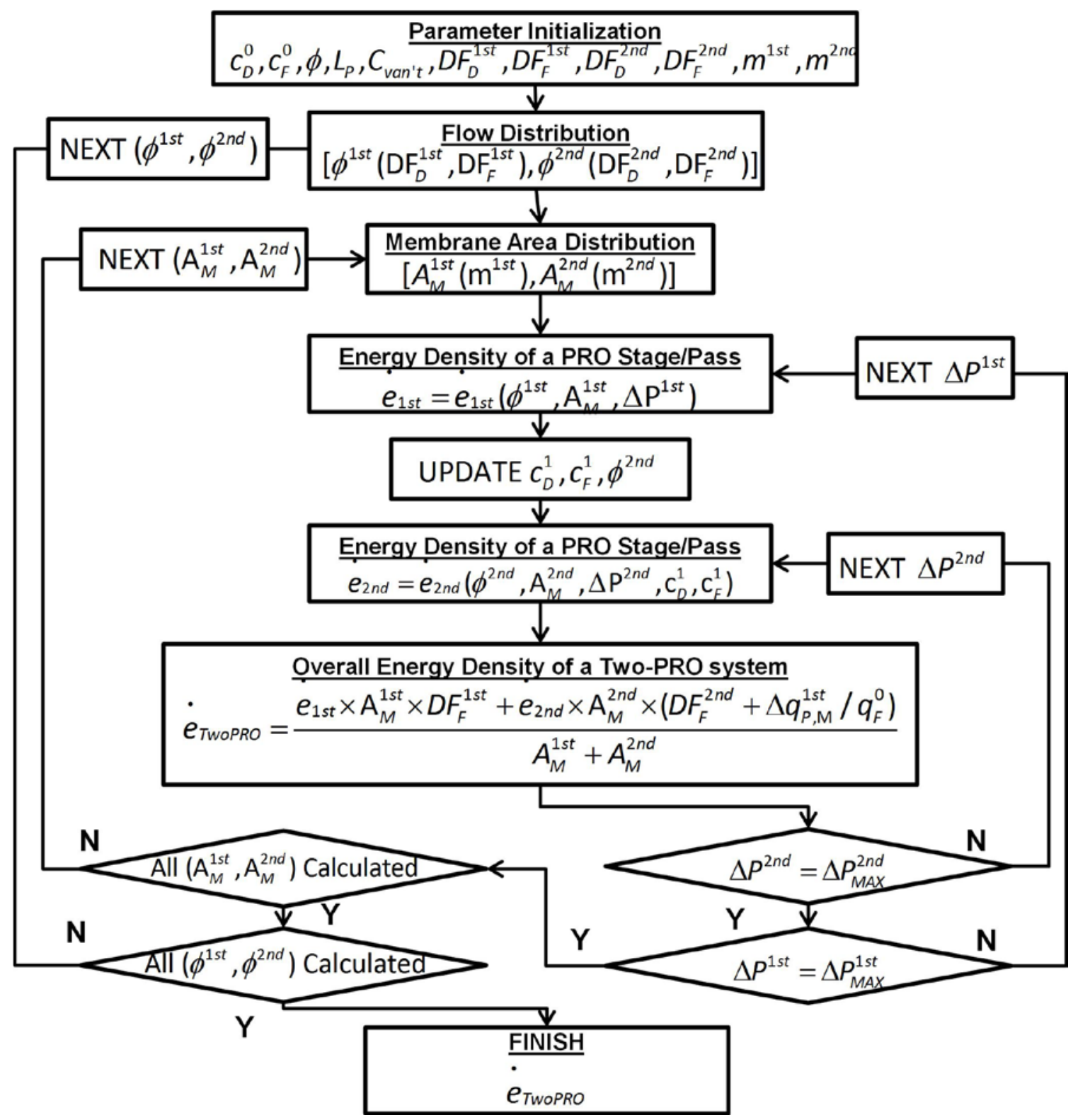

Fig. 11. Flowchart of the optimization of the average power density in the four configurations of the TwoPRO.

The scale-dependent average power density of the configuration CDCF is not as good as its scaleindependent salinity energy capacity. The relative average power density surplus is shown in Fig. 12 . The results indicate that generally at each dimensionless flow ratio, the overall membrane performance increases when more membrane is utilized. As seen in Fig. 12(a), (b), and (c), the relative average power density surplus significantly enhanced from $0.1 \mathrm{~m}^{2}$ to $0.4 \mathrm{~m}^{2}$. Moreover, comparing the results between different dimensionless flow ratios, the configuration CDCF shows advantageous average power density in higher dimensionless flow rates. With the dimensionless flow ratio 0.8 , all the four curves are above the zero line and reach higher values of the relative average power density surplus. Conversely, narrower range of advantageous operation and lower average power density surplus are achieved in Fig. 12(a) with $\phi=0.2$. 


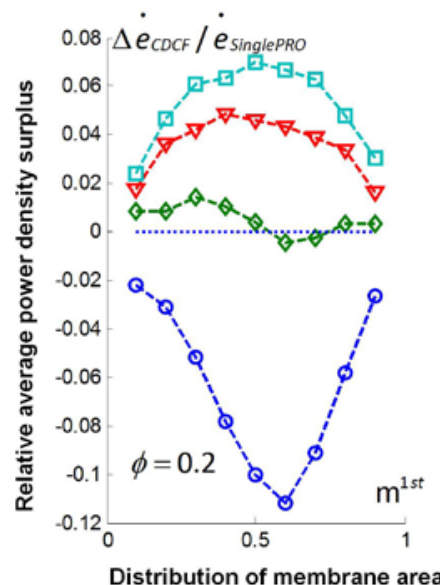

(a)

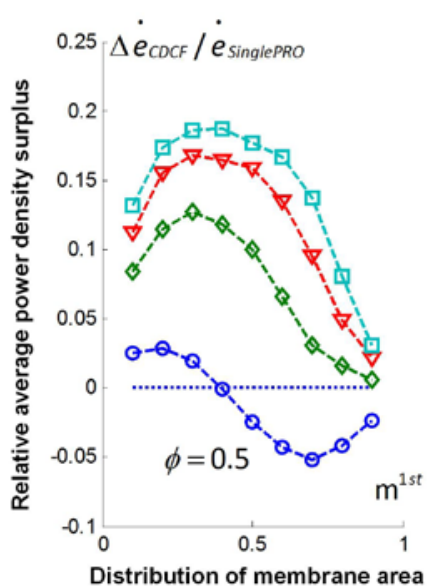

(b)

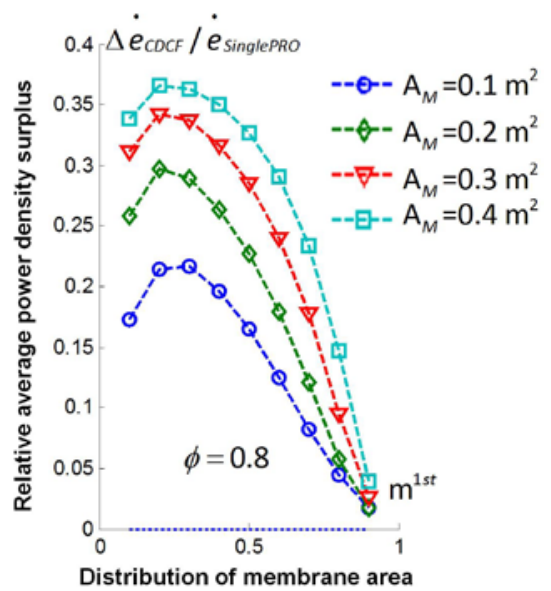

(c)

Fig. 12. Relative average power density surplus of the configuration CDCF.

For simplicity of visualization and the consistence of its energy capacity analysis in the configuration DDDF, the results shown in Fig. 13 are the optimum relative average power densities with respect to all the membrane allocation to each pair of the flow distribution rates of the DS and the FS. In (a), (b), (c) and (d), the total membrane usage of $0.1,0.2,0.3$ and $0.4 \mathrm{~m}^{2}$ are plotted, respectively.

\section{Relative average power density surplus of the configuration DDDF}

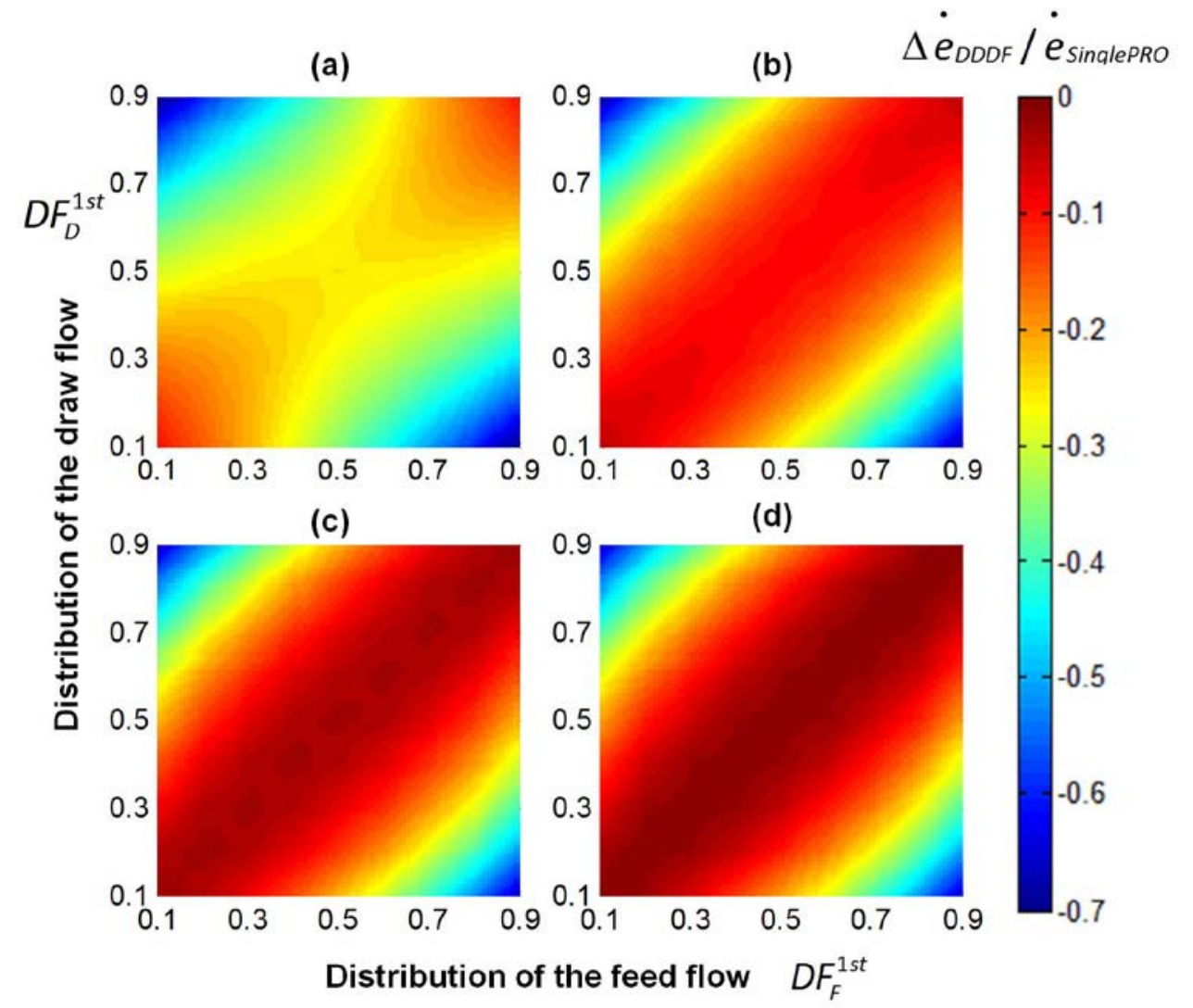

Fig. 13. Relative average power density surplus of the configuration DDDF. The relative average power density surplus of the DDDF with the total membrane usage of area $0.1,0.2,0.3$ and $0.4 \mathrm{~m}^{2}$ are presented in (a), (b), (c) and (d), respectively. 
The results show that the average power density of the configuration DDDF is also less than that of the optimum operation in the SinglePRO. When the dimensionless flow rate is 0.5 , all the relative energy surpluses in the four scales of the membrane area are negative. It means that the performance of the configuration DDDF is also worse than that of the SinglePRO from the perspective of overall membrane performance with respect to average power density.

The relative average power density surplus with respect to the feed flow distribution ratio and the membrane area allocation for the configuration CDDF are presented in Fig. 14. The four different membrane scales $\left(0.1,0.2,0.3\right.$ and $\left.0.4 \mathrm{~m}^{2}\right)$ and the three dimensionless flow rates $(0.2,0.5$ and 0.8$)$ are presented in (a)-(I), respectively. The results indicate the similar characteristics as that of the configuration CDCF that the overall membrane performance and advantageous operations are significantly improved when more area is utilized at all the dimensionless flow ratios. In Fig. 14, comparing the relative average power density surplus with the same dimensionless flow rate, such as results with $\phi=0.5$ shown in (e)-(h), the overall membrane performance is clearly improved from $0.1 \mathrm{~m}^{2}$ to $0.4 \mathrm{~m}^{2}$. In addition, with the same membrane area utilized, the higher dimensionless flow ratio raises the relative average power density. For instance, comparing the results shown in (c), (g) and $(\mathrm{k})$ where the membrane area is constantly $0.3 \mathrm{~m}^{2}$, with the dimensionless flow rate 0.8 , the overall membrane performance is considerably high. Moreover, compared with the average power density of the SinglePRO, it indicates that the configuration CDDF of the TwoPRO has better membrane performance with high membrane usage and high dimensionless rate. Also, advantageous operations (distribution flow ratios and membrane allocation ratios) can be improved and expanded when the dimensionless flow rate and the membrane area utilization increase.

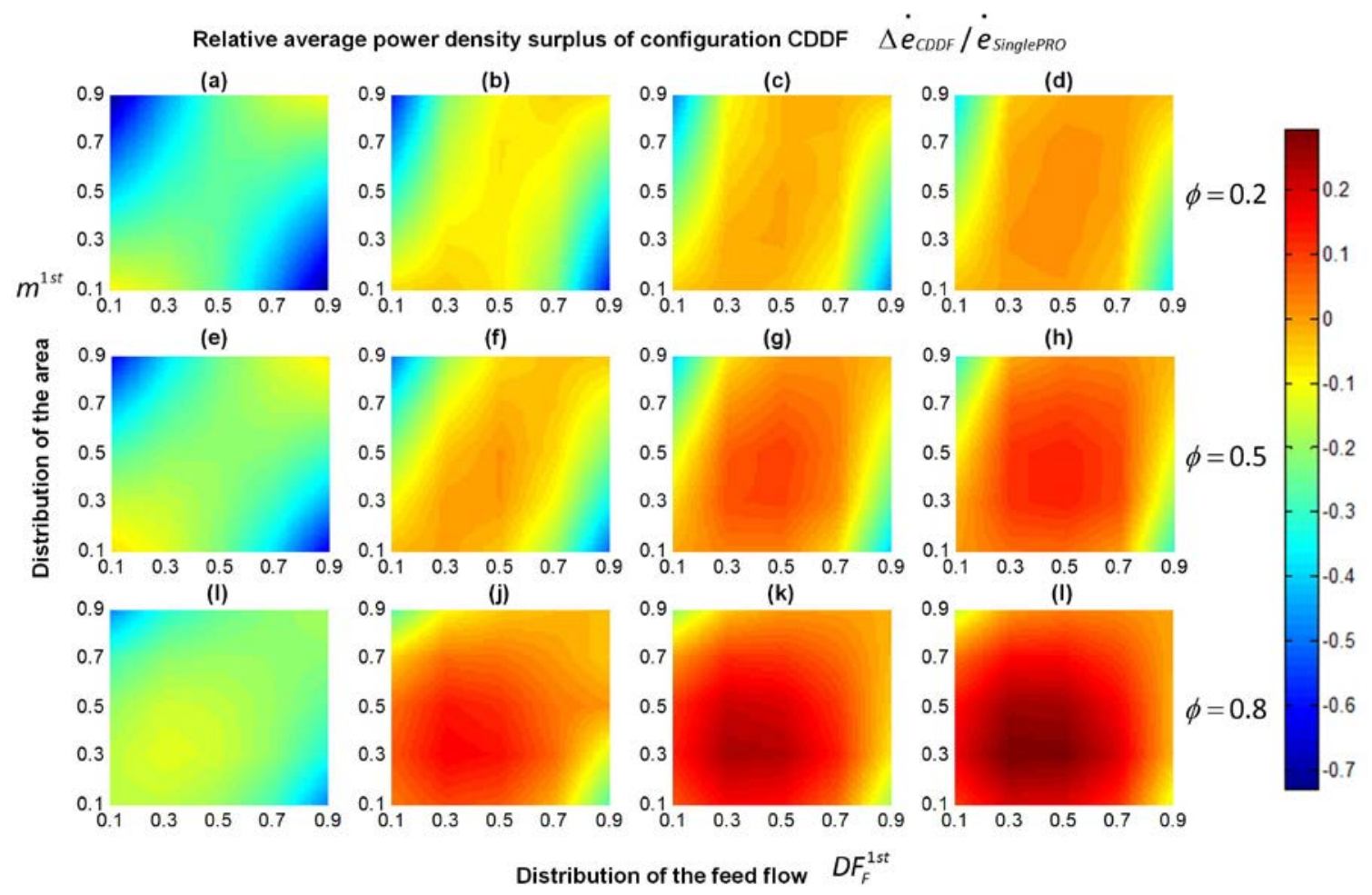

Fig. 14. Relative average power density surplus of the configuration CDDF. The configuration CDDF with different dimensionless flow rates and membrane usage are shown in (a)-(I), respectively. 
The relative average power density surplus of the configuration DDCF is presented in Fig. 15 in which three dimensionless flow rates, $0.2,0.5$ and 0.8 , and four scales of the membrane usage, 0.1 , $0.2,0.3$ and $0.4 \mathrm{~m}^{2}$, are included and shown in figures (a)-(I), respectively. However, the relative average power density of the configuration DDCF illustrated in Fig. 15 is not as encouraging as that of the configuration CDDF. Most of the average power density surpluses of all the three dimensionless flow rates and the four scales of membrane usage are lower than or close to zero. It is indicated from the overall membrane performance that the performance of the configuration DDCF is worse or similar to that of the SinglePRO. Furthermore, comparing the results between the configuration DDCF and CDDF, the role of the dimensionless flow rate in improving the energy density is different. In the configuration DDCF, with the same membrane usage, the higher average power density surplus is observed when the dimensionless flow rate is lower. For instance, comparing the results shown in (a), (e) and (I), the configuration with dimensionless flow rate 0.2 has the higher average power density.

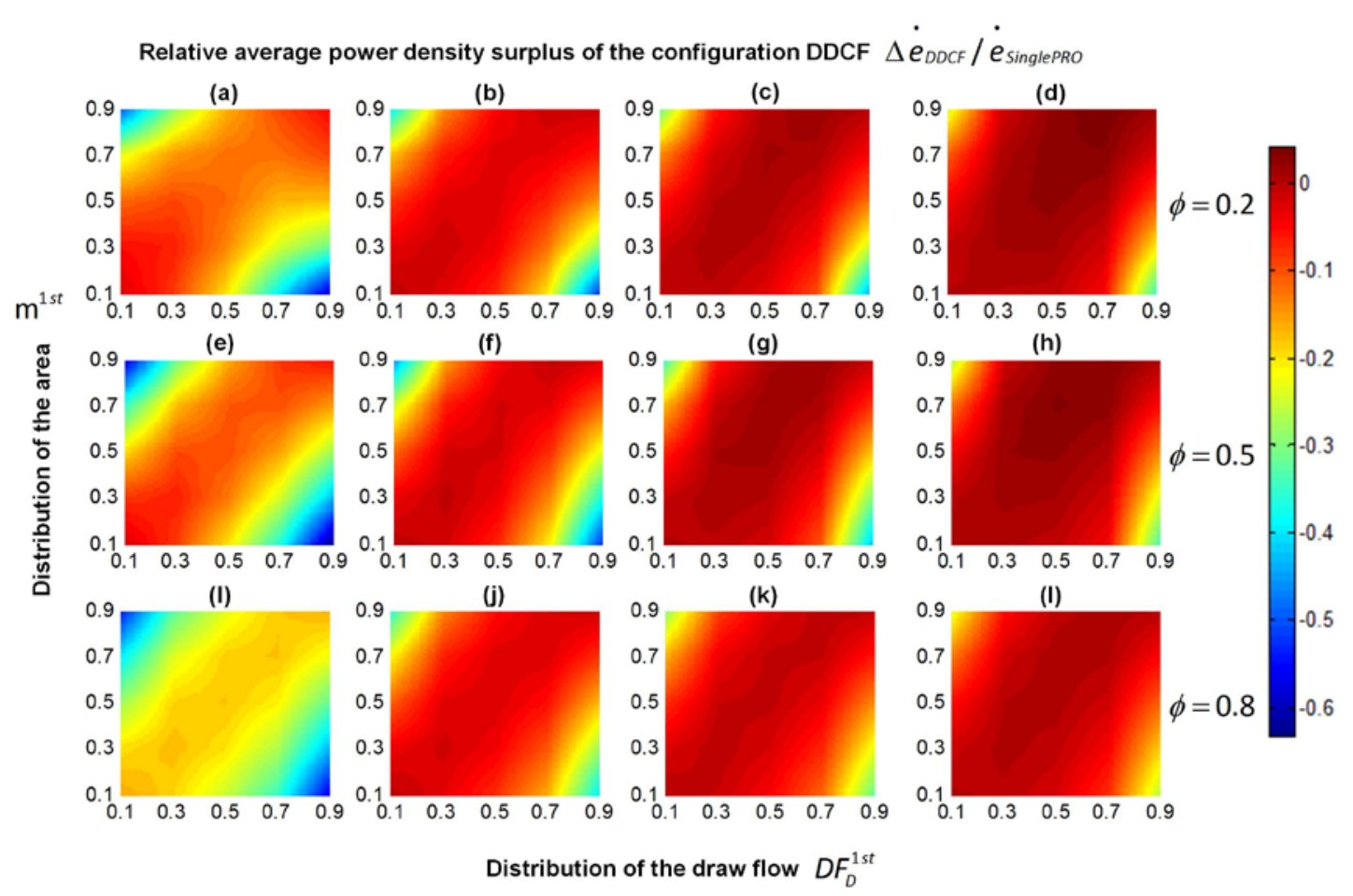

Fig. 15. The relative average power density surplus of the configuration DDCF. The configuration DDCF of the different dimensionless flow rates and membrane usages are shown in (a)-(I), respectively.

\section{Preference on TwoPRO or SinglePRO}

Based on the results of the system-based extractable energy above (Section 3.2), it is clear that the configurations CDCF and CDDF are superior to other configurations including the SinglePRO process. The energy surplus maxima of these two configurations are approximately achieved between dimensionless flow rate 0.5 and 0.6 in the configuration CDCF, and between 0.5 and 0.7 in the configuration CDDF. In such cases, the two configurations perform better than SinglePRO at the same dimensionless flow ratio. In addition, the average power density of the two configurations is also encouraging. With more area utilized in the PRO system, the advantage in the average power density of the TwoPRO strengthens. 
Preference between the TwoPRO and the SinglePRO is not as simple as it seems. Because if the salinity energy plant is considered at a fixed FS flow rate, analysis using the dimensionless flow ratio actually deals with different volumes of the salinity gradients. Consequently, the extractable energy capacity is different inherently. Taking the SinglePRO as an example, among the values of the extractable energy capacity and average power density of the dimensionless flow rate $0.2,0.5$ and 0.8 , a lower dimensionless flow rate means a significantly higher extractable energy capacity as illustrated in Fig. 3(b) and a wider feasible operation with certain satisfied membrane performance, e.g. $5 \mathrm{~W} \cdot \mathrm{m}^{-2}$ in Fig. 4(a). To this end, it may be argued that the improvement by applying the TwoPRO is the compensation of the less available energy caused by a high dimensionless flow rate.

However, PRO process at low dimensionless ratios is hardly implemented in practice because of two reasons. First, the dimensionless flow rate is generally determined by the local salinity gradients condition. In the coastal regions or estuaries, compared with the seawater, diluted stream rarely becomes the dominated source. In addition, the PRO process of the lower dimensionless flow ratios requires higher applied hydraulic pressure for the optimum operations (Fig. 3(c) and 4(b)), significantly challenging the current membrane technology. Therefore, it is more possible that a practical PRO process would be operated at a medium or little higher dimensionless flow rate. In such a case, several TwoPRO processes, i.e. CDCF and CDDF, are potentially beneficial from both the system-based energy capacity and the membrane performance. In addition, the advantageous TwoPRO configurations improve the permeation from the low concentration side to the high concentration side by the treatment in the two stages. To this end, the dilution of the draw solution is also strengthened. If the impaired water bodies, namely waste water and sewage, are used as the low concentration streams, the recycling efficiency of the impaired water can be increased by using the TwoPRO configurations. Furthermore, with the development of the high performance of the specific membrane for the PRO plant, the higher permeable membrane improves the efficiency of water permeation from the feed side. Similar to the thermodynamic restriction limit in RO, the equilibrium of the net driving force could be approximately built at the exit of the membrane module. In such a case, with the certain membrane usage, because of the high permeability, the scale of the permeation increases, then the advantageous performance of the TwoPRO configurations is also enhanced.

Currently, at the early stage of the practical PRO applications, even in SinglePRO, several main obstacles still remain to mitigate for wider implementation of PRO, such as the inefficiency of the pressure exchangers and hydro-turbines, and the lack of the high performance membrane. These potential problems also need to be addressed in a TwoPRO process. Based on the results of this study, the TwoPRO shows its advantageous performance at the locations with the high fraction of the high concentration salinity. With certain configurations, high efficiency of the energy extraction and the membrane usage is obtained by using a TwOPRO and the advantageous efficiency is further improved when more membrane is utilized in the salinity power extraction. In addition to the high effectiveness, the required optimum hydraulic pressures of the PRO plant with the higher dimensionless flow rates are lower which mitigates the requirement for the current membrane, indicating the potential applications in those suitable regions with high fraction of high salinities. Moreover, because TwoPRO configuration has more flexibility in the arrangement of the membrane modules, $\mathrm{ERD}(\mathrm{s})$ and $\mathrm{HT}(\mathrm{s})$, and the distribution of the DS and FS flow rates. It potentially provides alternative solution to improve the technology, which needs more investigations.

\section{Conclusions}


In this study, a systematic investigation of the TwoPRO is carried out in the potential extractable energy and membrane average power density. First, three key concepts of the PRO process; the power density, extractable energy and average power density are derived and analyzed in the case of a SinglePRO. Then, with the performance of the SinglePRO as the benchmark for comparisons, the TwoPRO system is analyzed. According to the flow schemes of the DS and FS, continuous or divided treatment, four configurations are proposed, which include CDCF that is continuous DS and continuous FS treatment, DDDF that is divided DS and divided FS treatment, CDDF that is continuous DS and divided FS treatment and DDCF that is divided DS and continuous FS treatment. Based on the optimum results obtained, some conclusions can be drawn: 1) for different objectives of the PRO optimization, the optimum operation of the hydraulic pressure on the draw solution are different; 2 ) from the perspective of the extractable energy, configurations CDCF, CDDF and DDCF have advantageous energy capacity compared to that of the SinglePRO; 3) from the membrane average power density perspective, the configurations CDCF and CDDF are potentially better at a high dimensionless flow rate. The advantage significantly strengthens when more membrane area is used in a TwoPRO.

\section{Nomenclature}

$A_{m}$

c

DF

$\mathrm{E}$

e

$\mathrm{L}_{p}$

m

$J_{w}$

$P$

$q$

$r$

W

$\pi$

$\phi$

Abbreviations

PRO
RO
HP
ERD
HT
BP
DS
FS
SinglePRO
TwoPRO
CDCF
DDDF

\author{
Membrane area $\left[\mathrm{m}^{2}\right]$ \\ Concentration of solution $\left[\mathrm{kg} \cdot \mathrm{m}^{-3}\right]$ \\ Distribution flow rate \\ Extractable energy [ $\mathrm{kWh} \cdot \mathrm{m}^{-3}$ ] \\ Membrane average power density $\left[\mathrm{W} \cdot \mathrm{m}^{-2}\right]$ \\ Membrane water permeability $\left[\mathrm{m}^{3} \cdot \mathrm{m}^{-2} \cdot \mathrm{h}^{-1} \cdot \mathrm{Pa}^{-1}\right]$ \\ Membrane area allocation rate \\ Water permeation flow rate $\left[\mathrm{m}^{3} \cdot \mathrm{m}^{-2} \cdot \mathrm{h}^{-1}\right]$ \\ Pressure $[\mathrm{Pa}]$ \\ Mass flow rate of solution $\left[\mathrm{m}^{3} \cdot \mathrm{h}^{-1}\right]$ \\ Relative average power density surplus \\ Membrane power density $\left[\mathrm{W} \cdot \mathrm{m}^{-2}\right]$ \\ Osmotic pressure $[\mathrm{Pa}]$ \\ Dimensionless flow rate
}


CDDF Continuous draw solution and divided feed solution

DDCF Divided draw solution and continuous feed solution

\section{Reference:}

[1] R.E. Pattle, Production of Electric Power by mixing Fresh and Salt Water in the Hydroelectric Pile, Nature, 174 (1954) 660-660.

[2] F. La Mantia, M. Pasta, H.D. Deshazer, B.E. Logan, Y. Cui, Batteries for Efficient Energy Extraction from a Water Salinity Difference, Nano Letters, 11 (2011) 1810-1813.

[3] N.Y. Yip, M. Elimelech, Performance Limiting Effects in Power Generation from Salinity Gradients by Pressure Retarded Osmosis, Environmental Science \& Technology, 45 (2011) 10273-10282.

[4] N.Y. Yip, M. Elimelech, Thermodynamic and Energy Efficiency Analysis of Power Generation from Natural Salinity Gradients by Pressure Retarded Osmosis, Environmental Science \& Technology, 46 (2012) 5230-5239.

[5] N.Y. Yip, M. Elimelech, Influence of Natural Organic Matter Fouling and Osmotic Backwash on Pressure Retarded Osmosis Energy Production from Natural Salinity Gradients, Environmental Science \& Technology, (2013).

[6] N.Y. Yip, A. Tiraferri, W.A. Phillip, J.D. Schiffman, L.A. Hoover, Y.C. Kim, M. Elimelech, Thin-Film Composite Pressure Retarded Osmosis Membranes for Sustainable Power Generation from Salinity Gradients, Environmental Science \& Technology, 45 (2011) 4360-4369.

[7] E. Brauns, Salinity gradient power by reverse electrodialysis: effect of model parameters on electrical power output, Desalination, 237 (2009) 378-391.

[8] R. Cusick, B. Bryan, D. Parker, M. Merrill, M. Mehanna, P. Kiely, G. Liu, B. Logan, Performance of a pilot-scale continuous flow microbial electrolysis cell fed winery wastewater, Appl Microbiol Biotechnol, 89 (2011) 2053-2063.

[9] R.D. Cusick, Y. Kim, B.E. Logan, Energy Capture from Thermolytic Solutions in Microbial ReverseElectrodialysis Cells, Science, 335 (2012) 1474-1477.

[10] J. Veerman, R.M. de Jong, M. Saakes, S.J. Metz, G.J. Harmsen, Reverse electrodialysis: Comparison of six commercial membrane pairs on the thermodynamic efficiency and power density, Journal of Membrane Science, 343 (2009) 7-15.

[11] P.M. Biesheuvel, A. van der Wal, Membrane capacitive deionization, Journal of Membrane Science, 346 (2010) 256-262.

[12] D. Brogioli, Extracting Renewable Energy from a Salinity Difference Using a Capacitor, Physical Review Letters, 103 (2009) 058501.

[13] D. Brogioli, R. Zhao, P.M. Biesheuvel, A prototype cell for extracting energy from a water salinity difference by means of double layer expansion in nanoporous carbon electrodes, Energy \& Environmental Science, 4 (2011) 772-777.

[14] D. Brogioli, R. Ziano, R.A. Rica, D. Salerno, F. Mantegazza, Capacitive mixing for the extraction of energy from salinity differences: Survey of experimental results and electrochemical models, Journal of Colloid and Interface Science, 407 (2013) 457-466.

[15] M. Mossad, L. Zou, A study of the capacitive deionisation performance under various operational conditions, Journal of Hazardous Materials, 213-214 (2012) 491-497.

[16] R. Rica, R. Ziano, D. Salerno, F. Mantegazza, R. van Roij, D. Brogioli, Capacitive Mixing for Harvesting the Free Energy of Solutions at Different Concentrations, Entropy, 15 (2013) 13881407.

[17] A. Achilli, A.E. Childress, Pressure retarded osmosis: From the vision of Sidney Loeb to the first prototype installation - Review, Desalination, 261 (2010) 205-211.

[18] G. Han, S. Zhang, X. Li, T.-S. Chung, High performance thin film composite pressure retarded osmosis (PRO) membranes for renewable salinity-gradient energy generation, J. Membr. Sci., 440 (2013) 108-121. 
[19] S.E. Skilhagen, Osmotic power - a new, renewable energy source, Desalination and Water Treatment, 15 (2010) 271-278.

[20] S.E. Skilhagen, J.E. Dugstad, R.J. Aaberg, Osmotic power - power production based on the osmotic pressure difference between waters with varying salt gradients, Desalination, 220 (2008) 476-482.

[21] S.-P. Sun, T.-S. Chung, Outer-Selective Pressure-Retarded Osmosis Hollow Fiber Membranes from Vacuum-Assisted Interfacial Polymerization for Osmotic Power Generation, Environmental Science \& Technology, (2013).

[22] G. Han, P. Wang, T.-S. Chung, Highly Robust Thin-Film Composite Pressure Retarded Osmosis (PRO) Hollow Fiber Membranes with High Power Densities for Renewable Salinity-Gradient Energy Generation, Environmental Science \& Technology, 47 (2013) 8070-8077.

[23] P. Sukitpaneenit, T.-S. Chung, High Performance Thin-Film Composite Forward Osmosis Hollow Fiber Membranes with Macrovoid-Free and Highly Porous Structure for Sustainable Water Production, Environmental Science \& Technology, 46 (2012) 7358-7365.

[24] M. Zhang, D. Hou, Q. She, C.Y. Tang, Gypsum Scaling in Pressure Retarded Osmosis: Experiments, Mechanisms and Implications, Water Research.

[25] Y. Xu, X. Peng, C.Y. Tang, Q.S. Fu, S. Nie, Effect of draw solution concentration and operating conditions on forward osmosis and pressure retarded osmosis performance in a spiral wound module, Journal of Membrane Science, 348 (2010) 298-309.

[26] Q. She, X. Jin, C.Y. Tang, Osmotic power production from salinity gradient resource by pressure retarded osmosis: Effects of operating conditions and reverse solute diffusion, Journal of Membrane Science, 401-402 (2012) 262-273.

[27] A.D. Wilson, F.F. Stewart, Deriving osmotic pressures of draw solutes used in osmotically driven membrane processes, Journal of Membrane Science, 431 (2013) 205-211.

[28] Y.C. Kim, M. Elimelech, Potential of osmotic power generation by pressure retarded osmosis using seawater as feed solution: Analysis and experiments, Journal of Membrane Science, 429 (2013) 330-337.

[29] J.R. McCutcheon, R.L. McGinnis, M. Elimelech, A novel ammonia-carbon dioxide forward (direct) osmosis desalination process, Desalination, 174 (2005) 1-11.

[30] H. Wei, W. Yang, M.H. Shaheed, Modelling and simulation of osmotic energy from salinity gradients: A case study from River Thames, in: Renewable Energy Research and Applications (ICRERA), 2013 International Conference on, 2013, pp. 907-912.

[31] Q.H. She, Y.K.W. Wong, S.F. Zhao, C.Y.Y. Tang, Organic fouling in pressure retarded osmosis: Experiments, mechanisms and implications, J. Membr. Sci., 428 (2013) 181-189.

[32] J.W. Post, J. Veerman, H.V.M. Hamelers, G.J.W. Euverink, S.J. Metz, K. Nymeijer, C.J.N. Buisman, Salinity-gradient power: Evaluation of pressure-retarded osmosis and reverse electrodialysis, J. Membr. Sci., 288 (2007) 218-230.

[33] K.S. Spiegler, Y.M. El-Sayed, The energetics of desalination processes, Desalination, 134 (2001) 109-128.

[34] T. Thorsen, T. Holt, The potential for power production from salinity gradients by pressure retarded osmosis, J. Membr. Sci., 335 (2009) 103-110.

[35] A. Zhu, P.D. Christofides, Y. Cohen, Effect of Thermodynamic Restriction on Energy Cost Optimization of RO Membrane Water Desalination, Industrial \& Engineering Chemistry Research, 48 (2008) 6010-6021.

[36] Y.W. Wei He, M. H. Shaheed, Energy and thermodynamic analysis of power generation using a natural salinity gradient based pressure retarded osmosis Desalination, (2014).

[37] A. Altaee, A. Sharif, G. Zaragoza, N. Hilal, Dual stage PRO process for power generation from different feed resources, Desalination, 352 (2014) 118-127.

[38] A. Achilli, T.Y. Cath, A.E. Childress, Power generation with pressure retarded osmosis: An experimental and theoretical investigation, Journal of Membrane Science, 343 (2009) 42-52. 
[39] Y.C. Kim, M. Elimelech, Adverse Impact of Feed Channel Spacers on the Performance of Pressure Retarded Osmosis, Environmental Science \& Technology, 46 (2012) 4673-4681.

[40] A.P. Straub, N.Y. Yip, M. Elimelech, Raising the Bar: Increased Hydraulic Pressure Allows Unprecedented High Power Densities in Pressure-Retarded Osmosis, Environmental Science \& Technology Letters, (2013).

[41] M. Elimelech, W.A. Phillip, The Future of Seawater Desalination: Energy, Technology, and the Environment, Science, 333 (2011) 712-717.

[42] Y.C. Kim, Y. Kim, D. Oh, K.H. Lee, Experimental Investigation of a Spiral-Wound PressureRetarded Osmosis Membrane Module for Osmotic Power Generation, Environmental Science \& Technology, 47 (2013) 2966-2973.

[43] A. Zhu, P.D. Christofides, Y. Cohen, Energy Consumption Optimization of Reverse Osmosis Membrane Water Desalination Subject to Feed Salinity Fluctuation, Industrial \& Engineering Chemistry Research, 48 (2009) 9581-9589.

[44] J.H. van't Hoff, The role of osmotic pressure in the analogy between solutions and gases, Journal of Membrane Science, 100 (1995) 39-44.

[45] E. Guggenheim, The theoretical basis of Raoult's law, Transactions of the Faraday Society, 33 (1937) 151-156.

[46] M.H. Sharqawy, L.D. Banchik, J.H. Lienhard V, Effectiveness-mass transfer units ( $\varepsilon-M T U$ ) model of an ideal pressure retarded osmosis membrane mass exchanger, Journal of Membrane Science, 445 (2013) 211-219.

[47] L.D. Banchik, M.H. Sharqawy, J.H. Lienhard V, Effectiveness-mass transfer units ( $\varepsilon$-MTU) model of a reverse osmosis membrane mass exchanger, Journal of Membrane Science, 458 (2014) 189-198.

[48] J.R. McCutcheon, M. Elimelech, Modeling water flux in forward osmosis: Implications for improved membrane design, AIChE Journal, 53 (2007) 1736-1744.

[49] J.R. McCutcheon, M. Elimelech, Influence of concentrative and dilutive internal concentration polarization on flux behavior in forward osmosis, Journal of Membrane Science, 284 (2006) 237247.

[50] W. He, Y. Wang, M.H. Shaheed, Modelling of osmotic energy from natural salt gradients due to pressure retarded osmosis: Effects of detrimental factors and flow schemes, Journal of Membrane Science, 471 (2014) 247-257.

[51] A.P. Straub, S. Lin, M. Elimelech, Module-Scale Analysis of Pressure Retarded Osmosis: Performance Limitations and Implications for Full-Scale Operation, Environmental Science \& Technology, (2014).

[52] L.D. Banchik, M.H. Sharqawy, J.H. Lienhard V, Limits of power production due to finite membrane area in pressure retarded osmosis, Journal of Membrane Science, 468 (2014) 81-89.

[53] W. He, Y. Wang, M.H. Shaheed, Energy and thermodynamic analysis of power generation using a natural salinity gradient based pressure retarded osmosis process, Desalination, 350 (2014) 86-94.

[54] L.D. Banchik, M.H. Sharqawy, J.H. Lienhard V, Limits of power production due to finite membrane area in pressure retarded osmosis, Journal of Membrane Science.

[55] M.H. Sharqawy, J.H. Lienhard, S.M. Zubair, Thermophysical properties of seawater: a review of existing correlations and data, Desalination and Water Treatment, 16 (2010) 354-380.

[56] A.P. Straub, N.Y. Yip, M. Elimelech, Raising the Bar: Increased Hydraulic Pressure Allows Unprecedented High Power Densities in Pressure-Retarded Osmosis, Environmental Science \& Technology Letters, 1 (2013) 55-59.

[57] V.t. Geraldes, V. Semião, M.N. de Pinho, Flow and mass transfer modelling of nanofiltration, Journal of Membrane Science, 191 (2001) 109-128. 